\title{
Optimal elastic coupling in form of one mechanical spring to improve energy efficiency of walking bipedal robots
}

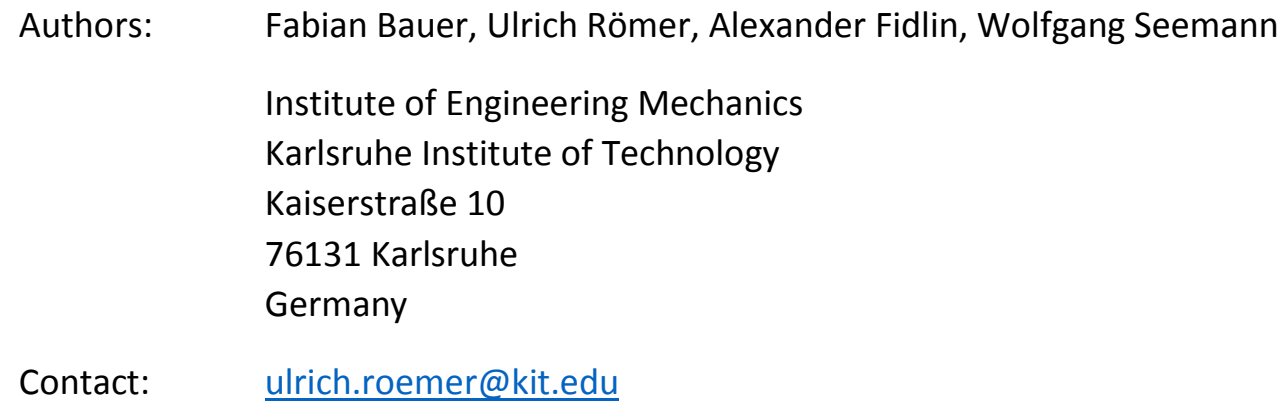

DOI: $\quad 10.1007 / \mathrm{s} 11044-016-9509-8$

The final publication is available at link.springer.com 


\title{
Optimal elastic coupling in form of one mechanical spring to improve energy efficiency of walking bipedal robots
}

\author{
Fabian Bauer · Ulrich Römer · Alexander Fidlin · \\ Wolfgang Seemann
}

Received: date / Accepted: date

\begin{abstract}
This paper presents a method to optimize the energy efficiency of walking bipedal robots by more than $80 \%$ in a speed range from 0.3 to $2.3 \mathrm{~m} / \mathrm{s}$ using elastic couplings - mechanical springs with movement speed independent parameters. The considered planar robot consists of a trunk, two two-segmented legs, two actuators in the hip joints, two actuators in the knee joints and an elastic coupling between the shanks. It is modeled as underactuated system to make use of its natural dynamics and feedback controlled via input-output linearization. A numerical optimization of the joint angle trajectories as well as the elastic couplings is performed to minimize the average energy expenditure over the whole speed range. The elastic couplings increase the swing leg motion's natural frequency thus making smaller steps more efficient which reduce the impact loss at the touchdown of the swing leg. The process of energy turnover is investigated in detail for the robot with and without elastic coupling between the shanks. Furthermore, the influences of the elastic couplings' topology and of joint friction are analyzed. It is shown that the optimization of the robot's motion and elastic coupling towards energy efficiency leads to a slightly slower convergence rate of the controller, yet no loss of stability but a lower sensitivity with respect to disturbances. The optimal elastic coupling discovered via numerical optimization is a linear torsion spring with transmissions between the shanks. A design proposal for this elastic coupling - which does not affect the robot's trunk and parallel shank motion and can be used to enhance an existing robot - is given for planar as well as spatial robots.
\end{abstract}

Keywords bipedal robot - dynamic walking - nonlinear feedback control · optimization . energy efficiency $\cdot$ elastic coupling

\section{Introduction}

There are many and varied application scenarios of bipedal robots. The most impressive ones are the humanoid as surrogate of workers in disaster response saving human lives and

F. Bauer · U. Römer $(\varangle) \cdot$ A. Fidlin $\cdot$ W. Seemann

Institute of Engineering Mechanics, Karlsruhe Institute of Technology, Kaiserstraße 10, 76131 Karlsruhe, Germany

E-mail: ulrich.roemer@kit.edu 
the exoskeleton as enhancement of disabled people improving human lives. These applications share inherently high mobility requirements, which prohibit an external power supply and therefore directly demand for high energy efficiency. The newly developed robot ATLAS from Boston Dynamics is employed in a disaster response scenario in the Robotics Challenge of the DARPA Program Maximum Mobility and Manipulation (M3). Its $23 \mathrm{~kg}$ lithium-ion-battery provides energy for no more than $20 \mathrm{~min}$ of running - the program consistently demands to improve the energy efficiency by a factor of 20 [36]. In order to evaluate the efficiency of locomotion, the dimensionless specific energetic cost of transport $c_{T}=$ energy input $/$ (weight $\times$ distance traveled) is applied. For example, the highly developed humanoid ASIMO from Honda $\left(c_{T}=3.2\right)$ has a 16 times higher specific cost of transport than a human $\left(c_{T}=0.2\right)$ at a low walking speed of $v=0.4 \mathrm{~m} / \mathrm{s}$ [13]. Due to this performance gap between artificial bipedal systems and their biological counterpart, energy efficiency can be justifiably stated as one of the major challenges in the development of walking bipedal robots [42].

There are essentially two reasons for the bad energy efficiency of todays bipedal robots. First the conservative control strategy: the robot is operated close to static equilibrium, fights against gravitation and tries to suppress any natural dynamics. Humans in contrast walk dynamically, they constantly fall over their feet, swinging their legs like pendulums during walking and therefore exploit their natural dynamics instead of struggling against them [31]. Second the design: the robot consists of rigid links interconnected only by actuated joints. Humans in contrast consist of both rigid skeletons and elastic structures serving as elastic boundary layer to absorb shocks, as pogo-stick to redirect center of mass motion and as return spring to buffer energy during the redirection of the swing leg motion [2].

Elastic boundary layers acting as shock absorbers are used in walking robots to reduce the load of the robots' mechanical parts, especially the actuator gear boxes [25, 26,29]. Because of their high stiffness compared to other elastic structures, they have a minor influence on the overall system dynamics.

Elastic couplings acting as pogo-stick have been used for a long time in hopping and running robots $[1,39]$ and their influence on the dynamics has been analyzed in detail with the model of the spring loaded inverted pendulum [6,9,19,22].

Elastic couplings acting as return springs have first been used solely in passive dynamic running [30,43]. Later an elastic coupling in the form of a hip spring was also used in spatial passive dynamic walking to adjust the step frequency to the frequency of the toddling motion for stabilization [27]. However, this is not a necessary measure [34]. The idea of adjusting the step frequency by a hip spring was also applied to prevent stumbling by shortening the swing phase of planar passive dynamic walking robots with trunk in simulation [46] and experiment [45].

By enhancing the simplest walking model $[3,21]$ with an elastic coupling in the form of a hip spring, the preferred speed-step length relationship of humans could be predicted [28]. With the same model it could be shown that at a given target speed the elastic coupling reduces the step length and therewith the impact loss at touchdown of the swing leg [17]. The robot Cornell Ranger used this effect to walk a marathon at one battery charge [7].

Elastic couplings acting as return springs have also been used in more complex walking models with segmented legs. For a passive model comprising of trunk, two thighs and two shanks it could be demonstrated that an elastic coupling between trunk and thigh is necessary for walking with upright trunk [8]. Using an elastic coupling over multiple joints, a trunkless model with impulsive push off force showed an increase in speed at constant energy input [16]. 
In addition there was the attempt to rebuild the anatomy of the biological example and its elastic couplings in detail. The robot FastRunner was inspired by an ostrich and uses a network of nonlinear elastic couplings with different modes for the swing phase and the stance phase of each leg. This allows for actuation of the six segmented leg by only one hip actuator [14]. In order to approach human locomotion capabilities, the robot BioBiped was designed [38]. However, its elastic couplings have only been investigated for a hopping motion with given joint torque trajectories without any optimization [37].

On the quest for an energy efficient bipedal robot a collision free gait on level ground was found with a planar passive robot model consisting of trunk, two rigid legs and two rotational springs between trunk and legs [23]. However, the gait without energy costs holds only for one specific speed, is unstable and comes along with a heavy oscillation of the trunk. On an incline this model has shown a much more stable gait than a comparable model without trunk [12].

A big step towards an energy efficient and versatile robot was made by enhancing this model with actuation in the hips [18,32]. Stiffness and resting length of hip springs were optimized together with the robot's motion. A significant reduction of actuation could be shown for one constant speed. However, both studies have three major drawbacks, which prevent their practical application: (1) the geometrical and inertial parameters of the investigated robots were far away from humanoid robots, (2) the objective function of optimization - integral of square of actuator torques over one step - has no physical relationship with energy used and does not account for variable step length and (3) the elastic couplings are optimized only for one specific speed whereas a real robot has to be energy efficient over a range of speeds.

In order to enable the practical applicability of elastic couplings in bipedal robots, these shortcomings are addressed. A process is developed to optimize the motion and the elastic couplings simultaneously over a broad range of speeds with respect to energy efficiency [5]. Furthermore a more realistic robot model comprising of the five rigid bodies trunk, thigh and shank close to the robots in $[11,24,40]$ is investigated. This allows for a thorough investigation of the effects of elastic couplings to develop an energy efficient robot [4].

This paper is organized as follows. Sec. 2 introduces the investigated robot model. It consists of the mechanics model (2.1), the actuator model (2.2) and the control strategy (2.3). Sec. 3 describes the process of generating the motion (3.1) as well as selecting the optimal elastic coupling (3.2) of the robot via simultaneous numerical optimization. Sec. 4 presents the effect of elastic couplings on the investigated robot. The reduction of specific cost of transport by elastic couplings is explained (4.1) and the influences of the elastic couplings' topology (4.2) and of joint friction (4.3) as well as stability and sensitivity of the motion with respect to disturbances (4.4) is displayed. Furthermore, a design proposal for the optimal elastic coupling is given (Sec. 4.5). Sec. 5 concludes the investigation, the used methods and the resulting insights and gives an outlook on the future research aims.

\section{Robot model}

The model of the investigated robot consists of the mechanics model (2.1), the actuator model (2.2) and the control strategy (2.3). The approach of modeling the robot as underactuated system and control it with input-output linearization was taken from [44]. 


\subsection{Mechanics model}

The robot is modeled as planar rigid body system. It is composed of two legs which are separately connected to a rigid trunk via two ideal revolute joints in the hip, whereas each leg consists of a rigid thigh and rigid shank also connected by a revolute joint as depicted in Fig. 1(a). The stance leg foot 1 is modeled a priori as ideal revolute joint and the swing leg foot 2 is assumed to move freely. It will be checked a posteriori, if the unilateral contact and stiction conditions are fulfilled. The configuration $\left(\mathbf{q}=\left[q_{1}, q_{2}, q_{3}, q_{4}, q_{5}\right]^{T}\right)$ of the robot is described by four joint angles $\left(\mathbf{q}_{J}=\left[q_{1}, q_{2}, q_{3}, q_{4}\right]^{T}\right)$ and one absolute orientation $\left(q_{A}=q_{5}\right)$. Two actuators between trunk and thigh and two actuators between thigh and shank (cf. Fig. 3(a)) apply torques $\left(\mathbf{u}=\left[u_{1}, u_{2}, u_{3}, u_{4}\right]^{T}\right)$ along the respective joint angles $\left(\mathbf{q}_{J}\right)$ and control their evolution. The dynamics of the multibody system can be described by

$$
\mathbf{M}(\mathbf{q}) \ddot{\mathbf{q}}+\mathbf{Q}(\mathbf{q}, \dot{\mathbf{q}})=\mathbf{B u}
$$

with the mass matrix $\mathbf{M}(\mathbf{q})$, the vector of generalized forces $\mathbf{Q}(\mathbf{q}, \dot{\mathbf{q}})$ and the input matrix B. The elastic couplings are introduced in Sec. 2.1.3, their restoring torques are included in the vector of generalized forces.

The later prescription of the joint angle trajectories $\left(\mathbf{q}_{J}\right)$ by the control (cf. Sec. 2.3) can be regarded as introduction of virtual constraints. No torques are applied about the point foot 1 or more precisely about the joint towards ground. Therefore, the evolution of the absolute orientation is subject to the remaining dynamics. The bipedal gait consists of two distinct phases which are distinguished by the number of feet on the ground. Adding this discrete state variable to the continuous state variables converts the system to a hybrid system.

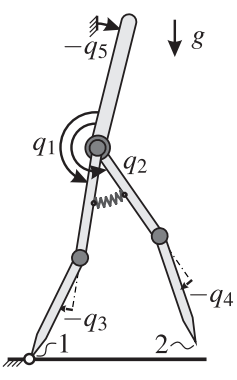

(a)

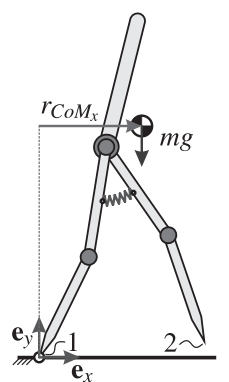

(b)

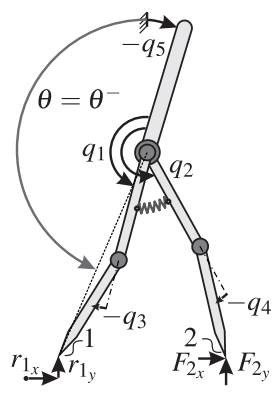

(c)

Fig. 1 Mechanics model of the robot (a) with single support phase as rotation of the total system about foot 1 (b) and double support phase as instantaneous impact of foot 2 (c)

\subsubsection{Single support phase}

During the single support phase solely foot 1 is on the ground. It corresponds to a rotation of the total robot about foot 1 powered by the torque due to the weight $r_{C_{O} M_{x}} m g$ about foot 1 as depicted in Fig. 1(b). The resulting motion can be described by the angular momentum theorem

$$
\dot{L}_{1}=-r_{\operatorname{CoM}_{x}}\left(\mathbf{q}_{J}, q_{A}\right) m g
$$


with the angular momentum $L_{1}$ of the total system about foot 1 . Since the trajectories of the joint angles $\mathbf{q}_{J}$ are prescribed by the control input $\mathbf{u}$, Eq. (2) corresponds to the remaining dynamics. In order to reflect this situation in the describing equations and to facilitate the control design, Eq. (1) is transformed into the mixed partial feedback linearized normal form. The mass matrix $\mathbf{M}$ does not depend on the absolute angle $q_{A}$ and the torques $\mathbf{u}$ do not directly act on it. Therefore, the equation of motion (1) can be subdivided into

$$
\left[\begin{array}{ll}
\mathbf{M}_{J J}\left(\mathbf{q}_{J}\right) & \mathbf{M}_{J A}\left(\mathbf{q}_{J}\right) \\
\mathbf{M}_{A J}\left(\mathbf{q}_{J}\right) & M_{A A}\left(\mathbf{q}_{J}\right)
\end{array}\right]\left[\begin{array}{c}
\ddot{\mathbf{q}}_{J} \\
\ddot{q}_{A}
\end{array}\right]+\left[\begin{array}{l}
\mathbf{Q}_{J}(\mathbf{q}, \dot{\mathbf{q}}) \\
Q_{A}(\mathbf{q}, \dot{\mathbf{q}})
\end{array}\right]=\left[\begin{array}{c}
\mathbf{B}_{J} \\
\mathbf{0}
\end{array}\right] \mathbf{u} .
$$

The angular acceleration of the absolute orientation

$$
\ddot{q}_{A}=-M_{A A}^{-1}\left(\mathbf{q}_{J}\right)\left(\mathbf{M}_{A J}\left(\mathbf{q}_{J}\right) \ddot{\mathbf{q}}_{J}+Q_{A}(\mathbf{q}, \dot{\mathbf{q}})\right)
$$

can be calculated with the second row of Eq. (3) and eliminated in the first row. Inverse dynamics determine the joint torques

$$
\begin{aligned}
\mathbf{u} & =\mathbf{B}_{J}^{-1}\left(\overline{\mathbf{M}}\left(\mathbf{q}_{J}\right) \mathbf{v}+\overline{\mathbf{Q}}(\mathbf{q}, \dot{\mathbf{q}})\right), \\
\overline{\mathbf{M}}\left(\mathbf{q}_{J}\right) & =\left(\mathbf{M}_{J J}\left(\mathbf{q}_{J}\right)-\mathbf{M}_{J A}\left(\mathbf{q}_{J}\right) M_{A A}^{-1}\left(\mathbf{q}_{J}\right) \mathbf{M}_{A J}\left(\mathbf{q}_{J}\right)\right), \\
\overline{\mathbf{Q}}(\mathbf{q}, \dot{\mathbf{q}}) & =\left(\mathbf{Q}_{J}(\mathbf{q}, \dot{\mathbf{q}})-\mathbf{M}_{J A}\left(\mathbf{q}_{J}\right) M_{A A}^{-1}\left(\mathbf{q}_{J}\right) Q_{A}(\mathbf{q}, \dot{\mathbf{q}})\right)
\end{aligned}
$$

as a function of the angular acceleration of the joint angles $\mathbf{v}=\ddot{\mathbf{q}}_{J}$, which becomes the new control input. Furthermore, the absolute orientation of the trunk $q_{A}$ is substituted by the absolute orientation of the virtual stance leg, the direct connection between hip and stance leg foot 1 (cf. Fig. 1(c))

$$
\boldsymbol{\theta}=q_{1}+\frac{1}{2} q_{3}+q_{5}=\mathbf{c}_{\theta} \mathbf{q},
$$

assuming equal segment length of thigh and shank. The canonical change of coordinates

$$
\tilde{\mathbf{q}}=\left[\begin{array}{c}
\mathbf{q}_{J} \\
\theta
\end{array}\right]=\left[\begin{array}{ll}
\mathbf{I} & \mathbf{0} \\
\mathbf{c}_{\theta}
\end{array}\right]\left[\begin{array}{l}
\mathbf{q}_{J} \\
q_{A}
\end{array}\right]=\mathbf{H q}
$$

transforms the mass matrix to

$$
\tilde{\mathbf{M}}\left(\mathbf{q}_{J}\right)=\left(\mathbf{H}^{-1}\right)^{T} \mathbf{M}\left(\mathbf{q}_{J}\right) \mathbf{H}^{-1} .
$$

The total angular momentum with respect to foot 1

$$
L_{1}=\left[\tilde{\mathbf{M}}_{A J}\left(\mathbf{q}_{J}\right) \tilde{M}_{A A}\left(\mathbf{q}_{J}\right)\right]\left[\begin{array}{c}
\dot{\mathbf{q}}_{J} \\
\dot{\theta}
\end{array}\right]
$$

is the generalized momentum conjugate to $\theta$ and can be determined directly with the mass matrix $\tilde{\mathbf{M}}$ and the angular velocities $\dot{\tilde{\mathbf{q}}}$. Hence, the system equation for the robot's stance phase in first order form can be displayed as

$$
\dot{\mathbf{x}}=\frac{\mathrm{d}}{\mathrm{d} t}\left[\begin{array}{c}
\mathbf{q}_{J} \\
\boldsymbol{\theta} \\
\dot{\mathbf{q}}_{J} \\
L_{1}
\end{array}\right]=\underbrace{\left[\begin{array}{c}
\dot{\mathbf{q}}_{J} \\
\tilde{M}_{A A}^{-1}\left(\mathbf{q}_{J}\right) L_{1}-\tilde{\mathbf{M}}_{A J}\left(\mathbf{q}_{J}\right) \dot{\mathbf{q}}_{J} \\
\mathbf{0} \\
-r_{C o M_{x}}\left(\mathbf{q}_{J}, \theta\right) m g
\end{array}\right]}_{\mathbf{f}(\mathbf{x})}+\underbrace{\left[\begin{array}{c}
\mathbf{0} \\
\mathbf{0} \\
\mathbf{I} \\
\mathbf{0}
\end{array}\right]}_{\mathbf{g}(\mathbf{x})} \mathbf{v}=: \mathbf{f}(\mathbf{x})+\mathbf{g}(\mathbf{x}) \mathbf{v}
$$

with $\overline{\mathbf{M}}_{A J}\left(\mathbf{q}_{J}\right)=\tilde{M}_{A A}^{-1}\left(\mathbf{q}_{J}\right) \tilde{\mathbf{M}}_{A J}\left(\mathbf{q}_{J}\right)$. 


\subsubsection{Double support phase}

During the instantaneous double support phase both feet are on the ground. It is modeled as plastic impact of foot 2 onto the ground with simultaneous contact opening in foot 1 (no interaction force: $\mathbf{F}_{1}=\mathbf{0}$ ), as depicted in Fig. 1(c). After the touchdown, foot 2 is at rest. It neither slips horizontally nor rebounds vertically. The configuration vector $\mathbf{q}_{a}=\left[\mathbf{q}^{T}, \mathbf{r}_{1}^{T}\right]^{T}$ is augmented by the position vector $\mathbf{r}_{1}$ of foot 1 to describe the double support phase. The impact duration is assumed to be infinitesimally short, therefore the configuration of the system

$$
\mathbf{q}_{a}^{+}=\mathbf{q}_{a}^{-}
$$

does not change over the impact, whereby ${ }^{-}\left({ }^{+}\right)$indicates the respective quantity before (after) the impact. The resulting motion can be described by the momentum theorem in integral form

$$
\mathbf{M}_{a}\left(\mathbf{q}_{a}^{-}\right) \dot{\mathbf{q}}_{a}^{+}-\mathbf{M}_{a}\left(\mathbf{q}_{a}^{-}\right) \dot{\mathbf{q}}_{a}^{-}=\hat{\mathbf{F}}_{a}=\lim _{t^{-} \rightarrow t^{+}} \int_{t^{-}}^{t^{+}} \mathbf{F}_{a}(t) \mathrm{d} t
$$

with the mass matrix $\mathbf{M}_{a}\left(\mathbf{q}_{a}\right)$ and the generalized impulse $\hat{\mathbf{F}}_{a}$ of the enhanced system. The generalized impulse

$$
\hat{\mathbf{F}}_{a}=\left(\frac{\partial \mathbf{r}_{2}\left(\mathbf{q}_{a}^{-}\right)}{\partial \mathbf{q}_{a}}\right)^{T} \hat{\mathbf{F}}_{2}
$$

can be expressed as projection of the impulse $\hat{\mathbf{F}}_{2}$ acting on foot 2 with the position vector $\mathbf{r}_{2}=\left[r_{2}, r_{2_{y}}\right]^{T}$ onto the configuration space. In combination with the condition for plastic impact $\dot{\mathbf{r}}_{2}\left(\mathbf{q}_{a}^{+}\right)=\mathbf{0}$, a system of linear equations can be formulated and solved for the generalized velocities after the impact and the impulse acting on foot 2 [44, pp. 55-57]

$$
\left[\begin{array}{c}
\dot{\mathbf{q}}_{a}^{+} \\
\hat{\mathbf{F}}_{2}
\end{array}\right]=\left[\begin{array}{c}
\boldsymbol{\Delta}_{\dot{\mathbf{q}}_{a}}\left(\mathbf{q}^{-}\right) \\
\boldsymbol{\Delta}_{\hat{\mathbf{F}}_{2}}\left(\mathbf{q}^{-}\right)
\end{array}\right] \dot{\mathbf{q}}^{-}, \quad \mathbf{q}_{a}^{-}=\left[\begin{array}{l}
\mathbf{I} \\
\mathbf{0}
\end{array}\right] \mathbf{q}^{-}, \quad \dot{\mathbf{q}}_{a}^{-}=\left[\begin{array}{c}
\mathbf{I} \\
\mathbf{0}
\end{array}\right] \dot{\mathbf{q}}^{-} .
$$

After the impact the role of the legs is switched, the former stance leg becomes the new swing leg and vice versa. Left and right leg are not distinguished, since only symmetrical gaits are investigated. The introduction of a switching matrix $\mathbf{R}$ which interchanges the legs' angles and angular velocities respectively allows for the mapping of the multibody state variables over the impact via

$$
\left[\begin{array}{c}
\mathbf{q}^{+} \\
\dot{\mathbf{q}}^{+}
\end{array}\right]=\left[\begin{array}{c}
\boldsymbol{\Delta}_{\mathbf{q}} \mathbf{q}^{-} \\
\boldsymbol{\Delta}_{\dot{\mathbf{q}}}\left(\mathbf{q}^{-}\right) \dot{\mathbf{q}}^{-}
\end{array}\right], \quad \boldsymbol{\Delta}_{\mathbf{q}}=\mathbf{R}, \quad \boldsymbol{\Delta}_{\dot{\mathbf{q}}}\left(\mathbf{q}^{-}\right)=[\mathbf{R} \mathbf{0}] \boldsymbol{\Delta}_{\dot{\mathbf{q}}_{a}}\left(\mathbf{q}^{-}\right)
$$

The angular momentum about foot 2 does not change during the impact

$$
L_{2}^{+}=L_{2}^{-} \text {. }
$$

By changing the reference point of the angular momentum from foot $1^{-}$to $2^{-}$just before the impact and switching the role of the legs from $2^{-}$to $1^{+}$just after the impact the angular momentum of the total system with respect to foot 1 is

$$
L_{1}^{+}=L_{1}^{-}-r_{2_{x}}\left(\mathbf{q}_{J}^{-}, \theta^{-}\right) m \frac{\mathrm{d} r_{C o M_{y}}\left(\mathbf{q}_{J}^{-}, \theta^{-}\right)}{\mathrm{d} t}
$$


In order to obtain a robust numerical process, the impact event - the end of the step and therefore the switching between the single support phase and the double support phase - is defined by the absolute orientation of the stance leg

$$
\mathscr{S}: \theta=\theta^{-}
$$

as depicted in Fig. 1(c). To guarantee physically reasonable results of the contact model, the conditions

$$
r_{2_{y}}\left(\mathbf{q}_{J}^{-}, \theta^{-}\right)=0
$$

for contact closing of foot 2 at the end of the step and

$$
\dot{r}_{2_{y}}\left(\mathbf{q}_{J}^{+}, \theta^{+}\right)>0
$$

for contact opening of foot 1 without interaction at the beginning of the step are introduced.

For both single and double support phase it has to be checked a posteriori, whether the solution fulfills the contact conditions unilaterality

$$
F_{1 y} \leq 0, \quad \hat{F}_{2_{y}} \leq 0,
$$

and stiction ${ }^{1}$

$$
\left|F_{1_{x}}\right| \leq \mu_{0}\left|F_{1_{y}}\right|, \quad \quad\left|\hat{F}_{2_{x}}\right| \leq \mu_{0}\left|\hat{F}_{2_{y}}\right|
$$

\subsubsection{Elastic couplings}

The investigated elastic couplings act with respect to the relative angle $\varphi$ between two bodies. Elastic couplings attached to the shank furthermore provide the option of weighting the knee joint angles $\left(q_{3}, q_{4}\right)$ in the relative angle $\varphi$ using a transmission ratio.

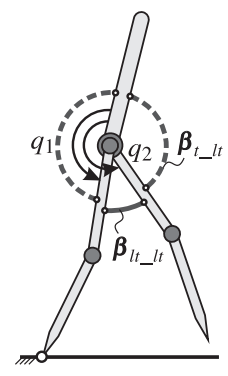

(a)

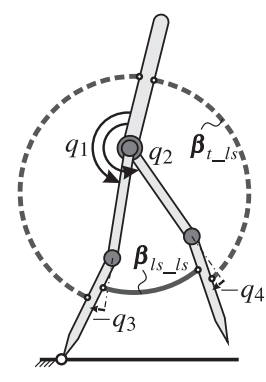

(b)

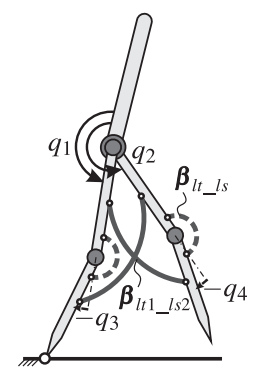

(c)

Fig. 2 Elastic coupling of the thighs $\left(l t \_l t, t \_l t\right)(\mathrm{a})$, the shanks $\left(l s \_l s, t \_l s\right)(\mathrm{b})$ and thigh and shank $\left(l t 1_{-} l s 2\right.$, $\left.l t \_l s\right)(\mathrm{c})$ divided into elastic couplings between the legs (solid line) and within each leg (dashed line)

There are six different elementary elastic couplings as depicted in Fig. 2. They can be subdivided into elastic couplings of the thighs ( $\left.l t_{-} l t, t_{-} l t\right)$ (cf. Fig. 2(a)), of the shanks

\footnotetext{
1 The coefficient of static friction $\mu_{0}$ is assumed to be the same for the continuous motion and the instance of impact. It has been observed that $\mu_{0}$ varies in the impact [10], which is neglected in this paper.
} 
$\left(l s \_l s, t \_l s\right)$ (cf. Fig. 2(b)) and of thigh and shank (lt1_ls2, lt_ls) (cf. Fig. 2(c)). For each configuration there is an elastic coupling between the legs (solid line) and within each leg (dashed line). The elastic couplings within each leg consist of two identical springs and correspond to the biological arrangement of elasticities in form of tendons.

The characteristics of each elastic coupling - the torque-angle-relationships - are described by a piecewise power law

$$
T_{e c}(\varphi)=\left\{\begin{array}{r}
-k\left(\varphi-\varphi_{0}\right)^{v}, \varphi-\varphi_{0} \geq 0 \\
k\left(\varphi_{0}-\varphi\right)^{v}, \varphi-\varphi_{0}<0
\end{array}\right.
$$

as approximation of a general nonlinear relationship. These characteristics can be realized via linear springs with geometric nonlinearities and end stops. The respective joint torque is calculated by

$$
\mathbf{T}_{J}(\varphi)=T_{e c}(\varphi) \nabla_{\mathbf{q}} \varphi
$$

with the torque angle relation $\left(T_{e c}\right)$ and the gradient of the relative angle $(\varphi)$ with respect to the configuration variables $(\mathbf{q})$ and is included in the generalized force $\mathbf{Q}(\mathbf{q}, \dot{\mathbf{q}})$ of Eq. (1).

The elastic coupling between trunk $(t)$ and thigh $(l t)$ acts on the relative angles $\varphi_{t_{-} l t 1}=$ $q_{1}$ and $\varphi_{t_{-} l t 2}=q_{2}$. It is defined by three parameters

$$
\boldsymbol{\beta}_{t_{-} l t}=\left[\varphi_{t_{-} l t_{0}}, k_{t_{-} l t}, v_{t_{-} l t}\right] .
$$

The elastic coupling between trunk $(t)$ and shank $(l s)$ acts on the relative angles $\varphi_{t_{-} l s 1}=$ $q_{1}+i_{t-l s} q_{3}$ and $\varphi_{t_{-} l s 2}=q_{2}+i_{t_{-} l s} q_{4}$. Knee joint angles and torques are transmitted with the ratio $i_{t} l s$. It is defined by four parameters

$$
\boldsymbol{\beta}_{t_{-} l s}=\left[\varphi_{t_{-} l s_{0}}, k_{t_{-} l s}, v_{t_{-} l s}, i_{t_{-} l s}\right] .
$$

The elastic coupling between thigh $(l t)$ and shank $(l s)$ acts on the relative angles $\varphi_{l t \_l s 1}=$ $q_{3}$ and $\varphi_{l t \_l s 2}=q_{4}$. It is defined by three parameters

$$
\boldsymbol{\beta}_{l t \_l s}=\left[\varphi_{l t_{-} l s_{0}}, k_{l t_{-} l s}, v_{l t_{-} l s}\right] .
$$

The elastic coupling between the thighs ( $l t)$ acts on the relative angle $\varphi_{l t} l t=q_{2}-q_{1}$. It has a vanishing resting angle $\varphi_{l t_{-} l_{0}}=0$, and is thus defined by two parameters

$$
\boldsymbol{\beta}_{l t_{-} l t}=\left[k_{l t} l t, v_{l t_{-} l t}\right] \text {. }
$$

The elastic coupling between the shanks $(l s)$ acts on the relative angle $\varphi_{l s_{-} l s}=q_{2}+$ $i_{l s_{-} l s} q_{4}-\left(q_{1}+i_{l s_{-} l s} q_{3}\right)$. Knee joint angles and torques are transmitted with the ratio $i_{l s_{-} l s}$ and the resting angle $\varphi_{l s_{-} l s_{0}}=0$ vanishes. Hence, it is defined by three parameters

$$
\boldsymbol{\beta}_{l s_{-} l s}=\left[k_{l s_{-} l s}, v_{l s_{-} l s}, i_{l s_{-} l s}\right] .
$$

The elastic coupling between the thigh (lt1) and the shank (lt2) of the opposite leg consists of two identical springs which act on the relative angles $\varphi_{l t 1 \_l s 2}=q_{2}+i_{l t 1 \_l s 2} q_{4}-q_{1}$ and $\varphi_{l t 1 \_l s 2}=q_{1}+i_{l t 1 \_l s 2} q_{3}-q_{2}$. Knee joint angles and torques are transmitted with the ratio $i_{l t 1 \_l s 2}$. It is defined by four parameters

$$
\boldsymbol{\beta}_{l t 1 \_l s 2}=\left[\varphi_{l t 1 \_l s 2_{0}}, k_{l t 1 \_l s 2}, v_{l t 1 \_l s 2}, i_{l t 1 \_l s 2}\right] .
$$

The general elastic coupling is a combination of the elementary elastic couplings. It is described by the parameters

$$
\boldsymbol{\beta}=\left[\boldsymbol{\beta}_{t \_l t}^{T}, \boldsymbol{\beta}_{t-l s}^{T}, \boldsymbol{\beta}_{l t \_l s}^{T}, \boldsymbol{\beta}_{l t \_l t}^{T}, \boldsymbol{\beta}_{l s \_l s}^{T}, \boldsymbol{\beta}_{l t 1 \_l s 2}^{T}\right]^{T} .
$$

These are physical parameters of the robot and therefore movement speed independent. The values of $\boldsymbol{\beta}$ are determined by numerical optimization as described in Sec. 3.2. 


\subsection{Actuator model}

Permanent magnet DC motors with transmission by cable and pulleys are selected as actuators as depicted in Fig. 3(b). The realization of the transmission by cable and pulleys

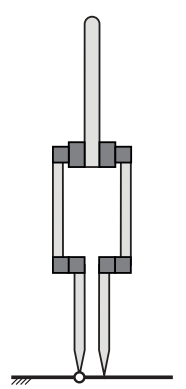

(a)

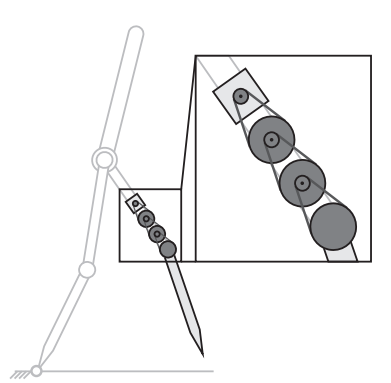

(b)

Fig. 3 Actuator installation circumstances (a) and realization by electric motor with cable and pulley transmission (b)

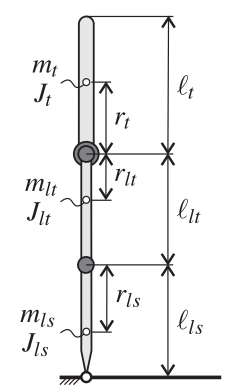

Fig. 4 Geometry and inertia parameters of the rigid body model

combined with a low transmission ratio $i_{T}$ leads to a low frictional resistance. Hence, the actuator is easily backdrivable. Under the assumption of a rigid drive train, the inertia of the actuator is reduced onto the inertia of the attached rigid body and the friction of the actuator is reduced onto viscous joint damping of the actuated joint with the coefficient of joint damping $d_{J}$. Due to the lower time constant in comparison to the mechanical dynamics of the robot, the electrical dynamics are neglected. The actuator remains an ideal torque source within a bounded torque range.

In order to quantify the used energy for walking, the electric power input of the actuator

$$
p_{A}=R_{A} i_{A}^{2}+k_{T} i_{A} \omega_{A} .
$$

is considered with resistance $R_{A}$, current $i_{A}$ and angular velocity $\omega_{A}$ of the armature and torque constant $k_{T}$. Employing the torque current relationship $T_{A}=k_{T} i_{A}$, the actuator-joint torque relationship $u=i_{T} T_{A}$ and the actuator-joint angular velocity relationship $\omega_{A}=i_{T} \dot{q}$ the electric power

$$
p_{A}=c_{s t a t} u^{2}+u \dot{q}, \quad c_{s t a t}=\frac{R_{A}}{\left(k_{T} i_{T}\right)^{2}}
$$

is displayed using merely variables of the rigid body model. The first term in Eq. (35) represents the electric power applied to produce a static torque without performing mechanical work. The second term represents the mechanical power. The coefficient of static electric power $c_{\text {stat }}$ determines how active power is distributed into dissipation power, which is lost as thermal output in the resistance of the armature and usable mechanical power. It is assumed that no electric energy can be recuperated during the operation of the electric motors in generator mode, since the electric circuit is not modeled and its efficiency is unknown. Hence, one actuator's energy consumption during one step

$$
W_{A}=\int_{0}^{T} \max \left(c_{s t a t} u^{2}+u \dot{q}, 0\right) \mathrm{d} t
$$

is calculated by integration of the positive electric power input over the step duration $T$. 


\subsection{Control}

The task of the controller is to realize a predefined motion of the robot by influencing the dynamics of the system. Therefore, the trajectories of the robot's joint angles $\mathbf{q}_{J}$ are controlled. The subsequently presented control approach is not developed by the authors but taken from [44].

\subsubsection{Reference trajectory}

The strictly decreasing orientation $\theta$ of the virtual stance leg, connecting hip and stance leg foot 1, (cf. Fig. 5) is used as independent variable of the reference trajectories' definition. Compared to time as independent variable this has the advantage of resulting in an
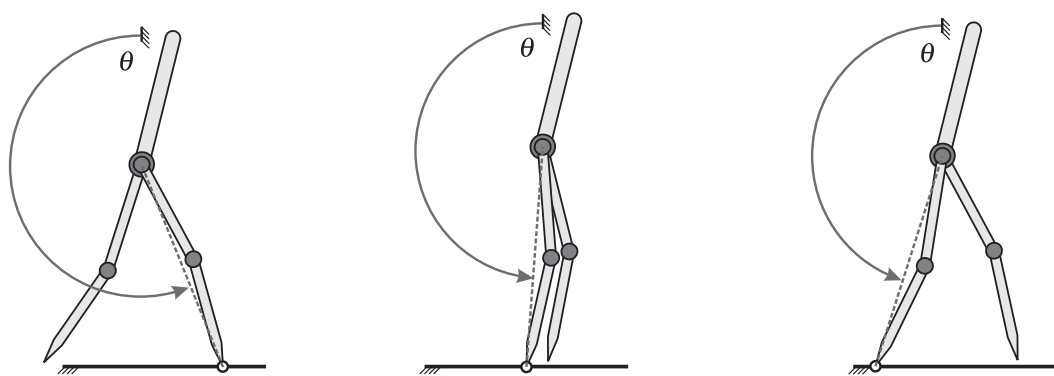

Fig. 5 Strictly decreasing orientation $\theta$ of the virtual stance leg, connecting hip and stance leg foot 1 , as independent variable for the reference trajectories' definition

autonomous system. The controller does not enforce the system's phase and the natural dynamics can evolve freely. The reference trajectories are discretized by means of a Bézier curve of degree $n_{\alpha}$

$$
\mathbf{h}_{r}(\theta)=\sum_{j=0}^{n_{\alpha}} \boldsymbol{\alpha}_{j}\left(\begin{array}{c}
n_{\alpha} \\
j
\end{array}\right)\left(\frac{\theta-\theta^{+}}{\theta^{-}-\theta^{+}}\right)^{j}\left(1-\frac{\theta-\theta^{+}}{\theta^{-}-\theta^{+}}\right)^{n_{\alpha}-j}
$$

normalized by the orientation of the virtual stance leg at the beginning $\theta^{+}$and end $\theta^{-}$of a step. The Bézier curve has a smoothening characteristic and contributes to a numerically robust process of the subsequent numerical optimization (cf. Sec. 3.1). Small changes in the parameters $\boldsymbol{\alpha}$ do not result in large oscillations of the Bézier curve [44, p. 139]. It allows for analytical expressions of function value and derivatives at the beginning $\left(\theta^{+}\right)$and the end $\left(\theta^{-}\right)$of a step

$$
\begin{aligned}
\mathbf{h}_{r}\left(\theta^{+}\right) & =\boldsymbol{\alpha}_{0}, & \frac{\partial \mathbf{h}_{r}}{\partial \theta}\left(\theta^{+}\right) & =\frac{n_{\alpha}\left(\boldsymbol{\alpha}_{1}-\boldsymbol{\alpha}_{0}\right)}{\theta^{-}-\theta^{+}}, \\
\mathbf{h}_{r}\left(\theta^{-}\right) & =\boldsymbol{\alpha}_{n_{\alpha}}, & \frac{\partial \mathbf{h}_{r}}{\partial \theta}\left(\theta^{-}\right) & =\frac{n_{\alpha}\left(\boldsymbol{\alpha}_{n_{\alpha}}-\boldsymbol{\alpha}_{n_{\alpha}-1}\right)}{\boldsymbol{\theta}^{-}-\boldsymbol{\theta}^{+}}
\end{aligned}
$$

as function of the Bézier coefficients $\boldsymbol{\alpha}_{i}$. Only periodical gaits with symmetrical steps of left and right foot are investigated. With the impact map Eq. (17) and

$$
\mathbf{q}=\mathbf{H}^{-1}\left[\begin{array}{c}
\mathbf{h}_{r}(\boldsymbol{\theta}) \\
\boldsymbol{\theta}
\end{array}\right], \quad \dot{\mathbf{q}}=\mathbf{H}^{-1}\left[\begin{array}{c}
\frac{\partial \mathbf{h}_{r}(\boldsymbol{\theta})}{\partial \theta} \\
1
\end{array}\right] \dot{\boldsymbol{\theta}}
$$


the Bézier parameters at the beginning of the step

$$
\begin{aligned}
{\left[\begin{array}{c}
\boldsymbol{\alpha}_{0} \\
\theta^{+}
\end{array}\right] } & =\mathbf{H} \boldsymbol{\Delta}_{\mathbf{q}} \mathbf{H}^{-1}\left[\begin{array}{c}
\boldsymbol{\alpha}_{n_{\alpha}} \\
\theta^{-}
\end{array}\right], \\
\boldsymbol{\alpha}_{1} & =[\mathbf{I} \mathbf{0}] \boldsymbol{\Delta}_{\dot{\mathbf{q}}} \mathbf{H}^{-1}\left[\begin{array}{c}
\boldsymbol{\alpha}_{n_{\alpha}}-\boldsymbol{\alpha}_{n_{\alpha}-1} \\
\frac{n_{\alpha}}{\theta^{-}-\theta^{+}}
\end{array}\right]\left(\mathbf{c}_{\theta} \boldsymbol{\Delta}_{\dot{\mathbf{q}}} \mathbf{H}\left[\begin{array}{c}
\frac{n_{\alpha}}{\theta^{-}-\theta^{+}}\left(\boldsymbol{\alpha}_{n_{\alpha}}-\boldsymbol{\alpha}_{n_{\alpha}-1}\right) \\
1
\end{array}\right]\right)^{-1}+\boldsymbol{\alpha}_{0}
\end{aligned}
$$

can be derived from the Bézier parameters at the end of the step $\left(\boldsymbol{\alpha}_{n_{\alpha}}, \boldsymbol{\alpha}_{n_{\alpha}-1}, \theta^{-}\right)$(cf. [44, pp. 141-143]). Hence, the reference trajectories of the joint angles and therefore the motion of the robot can be described by the Bézier parameters

$$
\boldsymbol{\alpha}=\left[\boldsymbol{\alpha}_{2}^{T}, \ldots, \boldsymbol{\alpha}_{n_{\alpha}}^{T}, \theta^{-}\right]^{T} .
$$

These describe the robot's motion and are therefore movement speed dependent. Their values are determined by numerical optimization as described in Sec. 3.1.

\subsubsection{Hybrid zero dynamics}

The control of the joint angles $\mathbf{q}_{J}$ is realized by input-output linearization. The system's output

$$
\mathbf{y}=\mathbf{h}(\mathbf{x})=\mathbf{h}_{r}(\boldsymbol{\theta})-\mathbf{q}_{J}
$$

is defined as the deviation of the joint angles from the reference trajectory. Differentiating the output twice with respect to time using chain rule and Eq. (12)

$$
\begin{aligned}
& \dot{\mathbf{y}}=\frac{\partial \mathbf{h}(\mathbf{x})}{\partial \mathbf{x}} \mathbf{f}(\mathbf{x})=: \mathrm{L}_{\mathbf{f}} \mathbf{h}(\mathbf{x}) \\
& \ddot{\mathbf{y}}=\frac{\partial \mathrm{L}_{\mathbf{f}} \mathbf{h}(\mathbf{x})}{\partial \mathbf{x}} \mathbf{f}(\mathbf{x})+\frac{\partial \mathrm{L}_{\mathbf{f}} \mathbf{h}(\mathbf{x})}{\partial \mathbf{x}} \mathbf{g}(\mathbf{x}) \mathbf{v}=: \mathrm{L}_{\mathbf{f}}^{2} \mathbf{h}(\mathbf{x})+\mathrm{L}_{\mathbf{g}} \mathrm{L}_{\mathbf{f}} \mathbf{h}(\mathbf{x}) \mathbf{v}
\end{aligned}
$$

relates it to the input $\mathbf{v}=\ddot{\mathbf{q}}_{J}$. A linear PD controller determines the behavior of the output's second derivative

$$
\ddot{\mathbf{y}}=-\mathbf{K}_{D} \dot{\mathbf{y}}-\mathbf{K}_{P} \mathbf{y}
$$

and achieves an asymptotically stable characteristic ${ }^{2}$ for

$$
\mathbf{y}=\mathbf{h}\left(\mathbf{q}_{J}, \theta\right)=0 .
$$

In the case of perfect joint angle reference trajectory tracking, the output as well as its time derivatives vanish $(\ddot{\mathbf{y}}=\mathbf{0})$. However, the system has remaining dynamics, the so called zero dynamics, corresponding to the rotation of the total system about foot 1 . The states of these dynamics are not observable by the output and define the zero dynamics manifold

$$
\mathscr{Z}=\{\mathbf{x} \mid \mathbf{y}=\mathbf{0}, \dot{\mathbf{y}}=\mathbf{0}\} .
$$

The zero dynamics are asymptotically stable, accomplished by the linear controller (cf. Eq. (47)), and invariant with respect to the impact $(\boldsymbol{\Delta}(\mathscr{S} \cap \mathscr{Z}) \subset \mathscr{Z})$ due to the design of the Bézier parameters (cf. Eq. (41) and (42)). The analysis of the multibody system can thus be reduced to the analysis of the hybrid zero dynamics ${ }^{3}$.

\footnotetext{
2 Successful implementations of real-time controllers based on input-output linearization and additional stabilization via linear PD control have been reported in literature, cf. [11,24,44].

3 Zero dynamics of a hybrid system are called hybrid zero dynamics.
} 
The single support phase of the hybrid zero dynamics is described by constraining Eq. (44) - (46) to

$$
\begin{aligned}
\mathbf{q}_{J} & =\mathbf{h}_{r}(\boldsymbol{\theta}) \\
\dot{\mathbf{q}}_{J} & =\frac{\partial \mathbf{h}_{r}(\boldsymbol{\theta})}{\partial \theta} \dot{\boldsymbol{\theta}}, \\
\mathbf{v}_{0} & =-\mathrm{L}_{\mathbf{g}} \mathrm{L}_{\mathbf{f}} \mathbf{h}(\mathbf{x})^{-1} \mathrm{~L}_{\mathbf{f}}^{2} \mathbf{h}(\mathbf{x})
\end{aligned}
$$

as well as the remaining dynamics from Eq. (12)

$$
\frac{\mathrm{d}}{\mathrm{d} t}\left[\begin{array}{c}
\theta \\
L_{1}
\end{array}\right]=\left[\begin{array}{c}
\tilde{M}_{A A}^{-1}\left(\mathbf{q}_{J}\right) L_{1}-\overline{\mathbf{M}}_{A J}\left(\mathbf{q}_{J}\right) \dot{\mathbf{q}}_{J} \\
-r_{C o M_{x}}\left(\mathbf{q}_{J}, \theta\right) m g
\end{array}\right]=\left[\begin{array}{c}
f_{\theta}(\theta) L_{1} \\
f_{L_{1}}(\theta)
\end{array}\right] .
$$

The special structure of Eq. (53) allows for a solution of the differential equation via quadrature as proposed in [44, pp. 128-131]. The time free formulation

$$
\frac{\mathrm{d} L_{1}}{\mathrm{~d} \theta}=\frac{f_{L_{1}}(\theta)}{f_{\theta}(\theta) L_{1}}
$$

is solved with separation of variables for the angular momentum ${ }^{4}$

$$
L_{1}(\theta)=-\sqrt{\left(L_{1}^{+}\right)^{2}+2 V_{0}(\theta)} .
$$

The term

$$
V_{0}(\theta)=\int_{\theta^{+}}^{\theta} \frac{f_{L_{1}}\left(\theta^{\prime}\right)}{f_{\theta}\left(\theta^{\prime}\right)} \mathrm{d} \theta^{\prime}
$$

as well as the time

$$
t(\theta)=\int_{\theta^{+}}^{\theta} \frac{1}{f_{\theta}\left(\theta^{\prime}\right) L_{1}\left(\theta^{\prime}\right)} \mathrm{d} \theta^{\prime}
$$

are determined via quadrature as functions of the orientation of the stance leg $\theta$.

The double support phase of the hybrid zero dynamics is described as linear mapping of the angular momentum over the impact

$$
L_{1}^{+}=\delta_{0}\left(\theta^{-}\right) L_{1}^{-}
$$

which follows from Eqs. (19) and (53). The factor $\delta_{0}$ only depends on the orientation of the stance leg at the end of the step and has to be determined only once for a specific set of reference trajectories $(\boldsymbol{\alpha})$.

\footnotetext{
4 Because the definition of $\theta$ is in mathematically positive direction it strictly decreases (cf. Fig. 5) and the angular momentum is negative.
} 
The gait consists of a sequence of single support phases and double support phases. It can be described by the Poincaré map

$$
P\left(L_{1}^{-}\right)=-\sqrt{\left(\delta_{0}\left(\theta^{-}\right) L_{1}^{-}\right)^{2}+2 V_{0}\left(\theta^{-}\right)},
$$

which maps the angular momentum $L_{1}^{-}$at the end of the step ${ }^{5}$ onto the end of the next step, using Eq. (58) in Eq. (56). Its fixed point states the periodic solution of the gait and is explicitly given by

$$
L_{1}^{-*}=-\sqrt{\frac{2 V_{0}\left(\theta^{-}\right)}{1-\delta_{0}\left(\theta^{-}\right)^{2}}} .
$$

The stability of the periodic solution $L_{1}^{-*}$ is evaluated by means of the absolute value of the Floquet multiplier, the eigenvalue of the monodromy matrix

$$
\left|\frac{\partial P\left(L_{1}^{-*}\right)}{\partial L_{1}^{-}}\right|=\delta_{0}\left(\theta^{-}\right)^{2}<1 .
$$

The sensitivity of the stable, periodical solution $L_{1}^{-*}$ is evaluated by means of the relative size

$$
s_{\mathscr{B}}=\frac{L_{1}^{-\max }-L_{1}^{-\min }}{L_{1}^{-*}}
$$

of its basin of attraction $\mathscr{B}$ which is bounded on both sides. The upper bound

$$
L_{1}^{-\max }=-\sqrt{-\frac{2}{\delta_{0}\left(\theta^{-}\right)^{2}} \min _{\theta^{+}<\theta<\theta^{-}} V_{0}(\theta)}
$$

ensures the forward rotation of the robot, the lower bound

$$
\begin{aligned}
& L_{1, F_{1 y}}^{-\min }=\inf \left\{L_{1}^{-} \mid \max _{\theta^{+}<\theta<\theta^{-}} F_{1_{y}}\left(\theta, L_{1}^{-}\right) \leq 0\right\}, \\
& L_{1}^{-\min }=\inf \left\{L_{1}^{-}>L_{1, F_{1 y}}^{-\min }\left|\max _{\theta^{+}<\theta<\theta^{-}}\right| \frac{F_{1_{x}}\left(\theta, L_{1}^{-}\right)}{F_{1_{y}}\left(\theta, L_{1}^{-}\right)} \mid \leq \mu_{0}\right\}
\end{aligned}
$$

ensures the contact conditions of unilaterality and stiction.

\subsection{Model parameters}

The model parameters used for the investigation of the influence of elastic couplings on the energy efficiency of bipedal robots are displayed in Table 1 and the process parameters in Table 2. In order to analyze effects relevant for practical systems, a $1.80 \mathrm{~m}$ tall and $80 \mathrm{~kg}$ heavy robot is investigated. The segmentation and distribution of mass is assumed to be human like. Therefore, the geometrical and inertial parameters (cf. Fig. 4) are determined via regression according to [15]. The rigid bodies' centers of mass, masses and moments of inertia values already include the inertia and reduced inertia of the actuators. The coefficient of static friction $\mu_{0}$ is estimated conservatively and assumed to include a safety margin

\footnotetext{
5 The touchdown of foot 2 defines the beginning of the step.
} 
Table 1 Model parameters

\begin{tabular}{llrl}
\hline \multicolumn{2}{l}{ Parameter } & Value & Unit \\
\hline$\ell$ & total body length & 1.80 & $\mathrm{~m}$ \\
$m$ & total body mass & 80.0 & $\mathrm{~kg}$ \\
$m_{t}$ & mass trunk & 48.2 & $\mathrm{~kg}$ \\
$m_{l t}$ & mass thigh & 11.3 & $\mathrm{~kg}$ \\
$m_{l s}$ & mass shank & 4.56 & $\mathrm{~kg}$ \\
$\ell_{t}$ & length trunk & 0.533 & $\mathrm{~m}$ \\
$\ell_{l t}$ & length thigh & 0.446 & $\mathrm{~m}$ \\
$\ell_{l s}$ & length shank & 0.446 & $\mathrm{~m}$ \\
$r_{t}$ & center of mass position trunk & 0.289 & $\mathrm{~m}$ \\
$r_{l t}$ & center of mass position thigh & 0.183 & $\mathrm{~m}$ \\
$r_{l s}$ & center of mass position shank & 0.267 & $\mathrm{~m}$ \\
$J_{t}$ & moment of inertia trunk & 3.09 & $\mathrm{~kg} \mathrm{~m}$ \\
$J_{l t}$ & moment of inertia thigh & 0.244 & $\mathrm{~kg} \mathrm{~m}$ \\
$J_{l s}$ & moment of inertia shank & 0.126 & $\mathrm{~kg} \mathrm{~m}$ \\
$g$ & standard gravity & 9.81 & $\mathrm{~m} / \mathrm{s}^{2}$ \\
$\mu_{0}$ & coefficient of static friction & 0.6 & - \\
$i_{T}$ & transmission ratio & $30: 1$ & - \\
$c_{s t a t}$ & coefficient of static power & $1.81 \mathrm{e}-3$ & $\mathrm{~W} /(\mathrm{Nm})^{2}$ \\
$d_{J}$ & reduced joint damping parameter & 8.00 & $\mathrm{Nm} \mathrm{s} / \mathrm{rad}$ \\
$n_{\alpha}$ & degree of Bézier curve & 21 & - \\
\hline
\end{tabular}

to cope with small disturbances of the reference motion ${ }^{6}$. The parameters concerning the actuators $\left(i_{T}, c_{s t a t}, d_{J}\right)$ are derived from the actuators of the walking robot MABEL [24,33]. The determined joint damping parameter $d_{J}$ is merely used for a realistic quantification of the specific cost of transport savings in Sec. 4.3. In the remainder of the paper, the joint damping is neglected. The selected degree of $n_{\alpha}=21$ for the Bézier curves is necessary because lower degrees were found to affect the results in Sec. 4.

\section{Optimization process}

In this section the generation of motion as well as the selection of elastic couplings by means of numerical optimization is described.

\subsection{Trajectory optimization}

In order to find an energy efficient motion, the trajectories of the joint angles $\mathbf{q}_{J}$ are optimized by determining the Bézier parameters $\boldsymbol{\alpha}$ for a specific movement speed. As objective function

$$
f_{\min _{\boldsymbol{\alpha}}}(\boldsymbol{\alpha}, \boldsymbol{\beta})=c_{T}=\frac{\sum_{i=1}^{4} \int_{0}^{T} \max \left(c_{s t a t} u_{i}^{2}+u_{i} \dot{q}_{i}, 0\right) \mathrm{d} t}{m g r_{2_{x}}\left(\theta^{-}\right)}
$$

the dimensionless specific cost of transport is selected, defined as energy input of the actuators (cf. Eq. (36)) divided by weight and step length.

\footnotetext{
6 Foot slipping does not immediately result in instability of the controller [35]. However, it is excluded from the following investigations in order to isolate the elastic couplings' effects from efficiency improvements due to slipping of the stance foot [20].
} 
Besides minimizing the objective function, the trajectory of the solution $\boldsymbol{\alpha}^{*}$ of the optimization has to fulfill equality and inequality constraints. The equality constraints $c_{i}(\boldsymbol{\alpha})=0$ are given by

$$
\begin{aligned}
\bar{v}-\frac{r_{2_{x}}\left(\theta^{-}\right)}{t\left(\theta^{-}\right)} & =0, \\
r_{2_{y}}\left(\theta^{-}\right) & =0
\end{aligned}
$$

and consist of the desired average horizontal velocity $\bar{v}$, in this paper referred to as speed, and contact closing at the end of the step (cf. Eq. (21)). The inequality constraints in the form $c_{i}(\boldsymbol{\alpha}) \leq 0$

$$
\begin{array}{rlrl}
F_{1_{y}} & \leq 0, & \hat{F}_{2_{y}} \leq 0, \\
\left|F_{1_{x}}\right|-\mu_{0}\left|F_{1_{y}}\right| & \leq 0, & \left|\hat{F}_{2_{x}}\right|-\mu_{0}\left|\hat{F}_{2_{y}}\right| \leq 0, \\
-\dot{r}_{2_{y}}\left(\theta^{+}\right) & \leq 0, & \\
-r_{2_{y}} & \leq 0, & & \\
q_{3} & \leq 0, & q_{4} \leq 0
\end{array}
$$

consist of unilaterality (cf. Eq. (23)), stiction (cf. Eq. (24)), contact opening without interaction (cf. Eq. (22)), no ground penetration of foot 2 during the step (cf. Eq. (21)) and no hyperextension of the knee joints. A SQP algorithm is utilized to solve the nonlinear, constrained optimization problem.

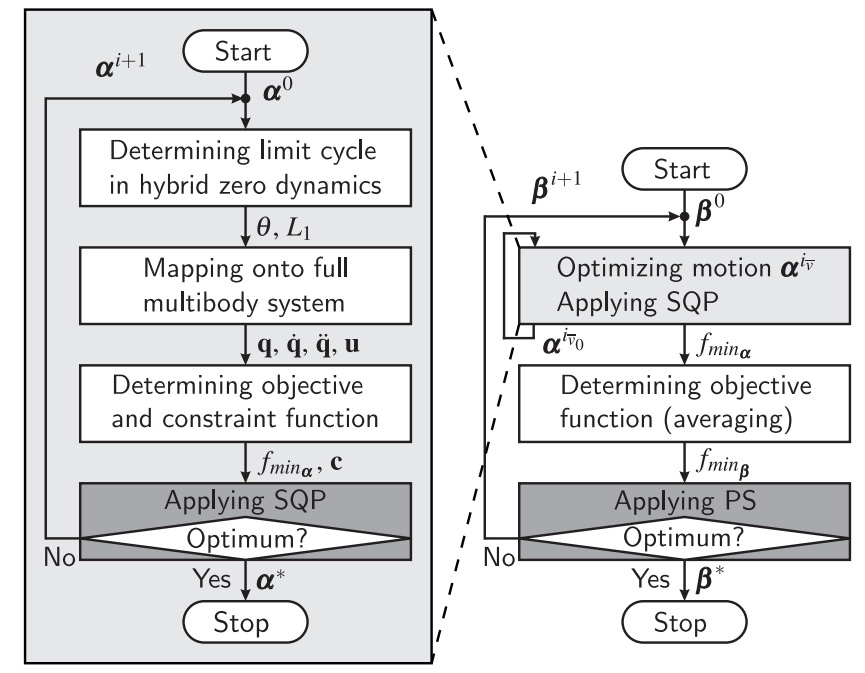

(a)

(b)

Fig. 6 Process of optimizing the motion $(\boldsymbol{\alpha})$ (a) and the elastic couplings $(\boldsymbol{\beta})$ (b)

Figure 6(a) depicts the process of optimizing the Bézier parameters $\boldsymbol{\alpha}$ implemented in MATLAB. For a given parameter set $\boldsymbol{\alpha}^{i}$ the limit cycle is determined in the hybrid zero dynamics via trapezoidal quadrature of Eq. (56) at $n_{\theta}$ sampling points and subsequent evaluation of the fixed point Eq. (60). Afterwards, the state variables of the hybrid zero dynamics 
Table 2 Process parameters

\begin{tabular}{lll}
\hline \multicolumn{2}{c}{ Parameter } & Value \\
\hline$n_{\theta}$ & Number of quadrature sampling points & 401 \\
TolFun & SQP algorithm termination function tolerance & $1 \mathrm{e}-2$ \\
TolCon & SQP algorithm termination constraint tolerance & $1 \mathrm{e}-5$ \\
$n_{\bar{v}}$ & Number of averaging sampling points & 10 \\
TolMesh & PS algorithm termination mesh tolerance & $1 \mathrm{e}-5$ \\
\hline
\end{tabular}

$\left(\theta, L_{1}\right)$ are mapped onto the state variables and angular accelerations of the full multibody system (q, $\mathbf{q}, \ddot{\mathbf{q}})$ by using Eq. (50) - (52) and (4). The respective joint torque $\mathbf{u}$ is determined by evaluation of Eq. (5). The objective function is evaluated via trapezoidal quadrature of Eq. (66) at $n_{\theta}$ sampling points with linear interpolation of zero crossings. The constraint function is also evaluated at $n_{\theta}$ sampling points. Objective and constraint function are forwarded to MATLAB's implementation of the SQP algorithm, which calculates the parameter set $\boldsymbol{\alpha}^{i+1}$ for the next iteration using a quasi Newton approach. The optimum described by the parameter set $\boldsymbol{\alpha}^{*}$ is reached, if the termination tolerance for the function TolFun and for the constraints TolCon are fulfilled. The termination tolerances in Table 2 were chosen instead of the default parameters because of convergence difficulties due to gradient approximations via finite differences ${ }^{7}$. At this point it has to be mentioned, that there is no iteration in the determination of the limit cycle neither in the full multibody system nor in the hybrid zero dynamics. The limit cycle is given directly by Eq. (60). Hence, this approach is numerically very efficient ${ }^{8}$ and allows for the optimization of the elastic couplings in an outer loop.

\subsection{Structure optimization}

While the Bézier parameters $\boldsymbol{\alpha}$ are changed and optimized separately for each speed $\bar{v}$, the structure and therefore the parameters of the elastic couplings $\boldsymbol{\beta}$ are fixed after design and independent of movement speed. Hence, they have to be a trade-off for different speeds. In order to find an energy efficient robot in a broad region of operation, the elastic couplings $\boldsymbol{\beta}$ are optimized for the speed range $\bar{v}_{i} \in[0.3,2.3] \mathrm{m} / \mathrm{s}$. As objective function

$$
f_{\min _{\boldsymbol{\beta}}}(\boldsymbol{\beta})=\bar{c}_{T}=\frac{1}{n_{\bar{v}}} \sum_{i_{\bar{v}}=1}^{n_{\bar{v}}} f_{\min _{\boldsymbol{\alpha}}}\left(\boldsymbol{\alpha}^{i_{\bar{v}}}, \boldsymbol{\beta}\right)
$$

the mean specific cost of transport for the speed range is selected. Whereas the interval $[0.3,2.3] \mathrm{m} / \mathrm{s}$ is divided into $n_{\bar{v}}$ subintervals which are evaluated at their midpoints.

Figure 6(b) depicts the process of optimizing the elastic coupling parameters $\boldsymbol{\beta}$ implemented in MATLAB. For a given parameter set $\boldsymbol{\beta}^{i}$ the motion $\boldsymbol{\alpha}^{i_{\bar{v}}}$ is optimized for $n_{\bar{v}}$ single speeds $\bar{v}_{i}$ separately. The optimization of motion is performed in an inner loop displayed in Fig. 6(a). The objective function is determined by averaging the single specific costs of transport of the different speeds. Since the process of optimizing motion adds numerical

\footnotetext{
7 Convergence can be improved by a more accurate numerical approximation of gradients, e.g. via complex step derivative approximation [41] which was investigated after submission of this paper.

8 Computation times strongly depend on the parameters in Tables 1 and 2 as well as the initial conditions. Our implementation requires about 10 s to find the optimal trajectory parameters $\boldsymbol{\alpha}$ when initialized with the solution for a $0.2 \mathrm{~m} / \mathrm{s}$ faster speed. The computations were performed on a HP Z600 workstation with two Intel Xeon X5650 processors.
} 
noise, the optimization problem is non-smooth and nonlinear. It is solved by a gradient free direct search algorithm called pattern search. The approach varies each parameter consecutively around the reference point $\boldsymbol{\beta}^{i}$ by steps $\Delta \boldsymbol{\beta}$ of equal magnitude. The point in the mesh with the lowest value becomes the new reference point, if the new point equals the old point, the mesh size will be halved until the pattern search termination mesh size tolerance TolMesh is reached.

\section{Results}

In this section, the effect of elastic couplings on the robot is presented. The investigation focuses on the reduction of the specific cost of transport. The robot's motion and energy turnover are analyzed to identify the major energy loss component as well as to suggest counter measures (Sec. 4.1). Subsequently the influence of the elastic couplings' topology (Sec. 4.2) and of joint damping (Sec. 4.3) are investigated focusing on the reduction of the specific cost of transport. Furthermore, the influence of the optimal elastic coupling on stability and sensitivity with respect to disturbances of the robot's motion is analyzed (Sec. 4.4). Finally, a design proposal for the optimal elastic coupling is presented in Sec. 4.5.

\subsection{Reduction of cost of transport}

In order to isolate the effect of elastic couplings, a robot without elastic couplings is considered first. Its motion $(\boldsymbol{\alpha})$ is optimized for each speed by the process described in Sec. 3.1. Figure 7 displays the resulting motions for the lowest $(\bar{v}=0.3 \mathrm{~m} / \mathrm{s})$, medium $(\bar{v}=1.3 \mathrm{~m} / \mathrm{s})$ and highest $(\bar{v}=2.3 \mathrm{~m} / \mathrm{s})$ considered speed at seven equidistant instances of time.

The step length, the trunk inclination angle and the stance leg knee flexion angle of the robot increase with increasing speed. For example, the maximum trunk inclination angle increases from $1.09^{\circ}$ at $\bar{v}=0.3 \mathrm{~m} / \mathrm{s}$ to $24.5^{\circ}$ at $\bar{v}=2.3 \mathrm{~m} / \mathrm{s}$. In order to investigate the energy turnover of the robot, the specific energies ${ }^{9}$

$$
\begin{aligned}
c_{T} & =\frac{\sum_{i=1}^{4} \int_{0}^{T} \max \left(c_{\text {stat }} u_{i}^{2}+u_{i} \dot{q}_{i}, 0\right) \mathrm{d} t}{m g r_{2_{x}}\left(\theta^{-}\right)} \\
e_{\text {mech }}^{+} & =\frac{\sum_{i=1}^{4} \int_{0}^{T} \max \left(u_{i} \dot{q}_{i}, 0\right) \mathrm{d} t}{m g r_{2_{x}}\left(\theta^{-}\right)} \\
e_{\text {mech }}^{-} & =-\frac{\sum_{i=1}^{4} \int_{0}^{T} \min \left(u_{i} \dot{q}_{i}, 0\right) \mathrm{d} t}{m g r_{2_{x}}\left(\theta^{-}\right)} \\
e_{\text {imp }} & =-\frac{E_{k i n}\left(\theta^{+}\right)-E_{k i n}\left(\theta^{-}\right)}{m g r_{2_{x}}\left(\theta^{-}\right)} \\
e_{\text {stat }} & =\frac{\sum_{i=1}^{4} \int_{0}^{T} c_{\text {stat }} u_{i}^{2} \mathrm{~d} t}{m g r_{2_{x}}\left(\theta^{-}\right)}
\end{aligned}
$$

are introduced. Analogously to the specific cost of transport $c_{T}$, these quantities are related to the robots weight $m g$ and the distance traveled which is the step length $r_{2_{x}}\left(\theta^{-}\right)$. While the specific cost of transport $c_{T}$ corresponds to the electric energy intake of the actuators, the

\footnotetext{
9 In this paper the term specific energies refers to the quantities introduced in Eq. (75) - Eq. (79) although some of them are works (process, not state quantities).
} 


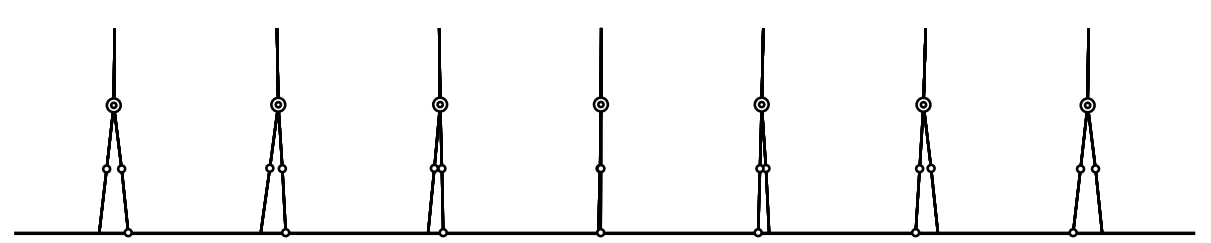

(a) $\bar{v}=0.3 \mathrm{~m} / \mathrm{s}$

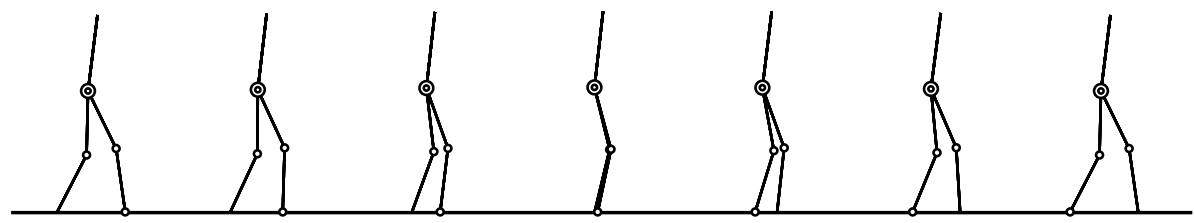

(b) $\bar{v}=1.3 \mathrm{~m} / \mathrm{s}$

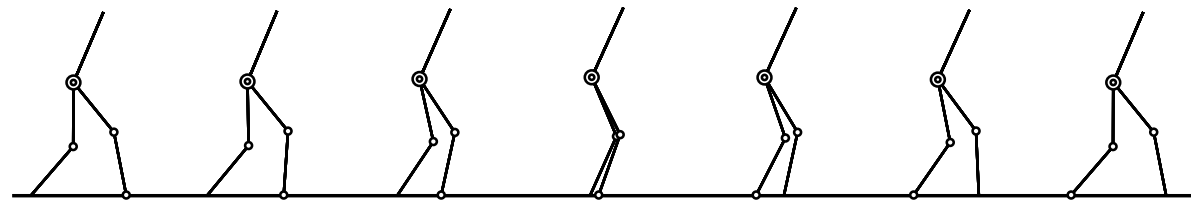

(c) $\bar{v}=2.3 \mathrm{~m} / \mathrm{s}$

Fig. 7 Comparison of the robot's motion without elastic coupling at three different speeds $\bar{v}$ at seven equidistant instances of time

specific positive mechanical work $e_{\text {mech }}^{+}$corresponds to the mechanical energy input in motor or accelerating mode and the specific negative mechanical work $e_{\text {mech }}^{-}$to the mechanical energy takeout in generator or breaking mode. The specific impact loss $e_{i m p}$ is defined by the loss of kinetic energy during the impact. The specific static energy $e_{\text {stat }}$ is defined by the heat loss in the resistance of the actuator's armature and corresponds to the electric energy effort necessary to statically supply a torque.

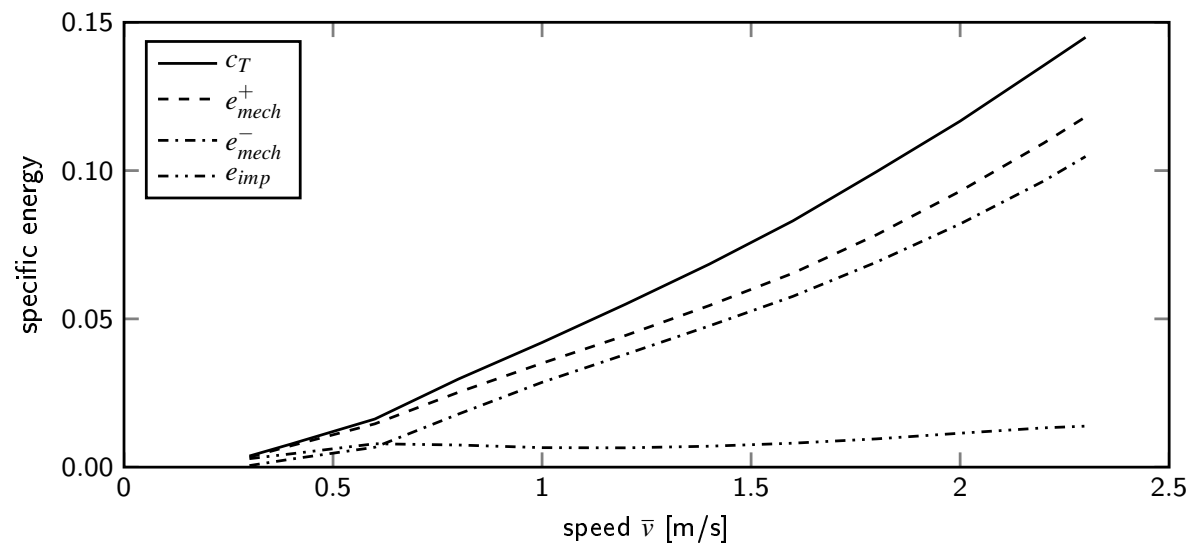

Fig. 8 Comparison of the specific energies of the robot without elastic coupling 
Figure 8 illustrates the specific energies of the robot without elastic coupling. The specific cost of transport $c_{T}$ increases with speed $\bar{v}$ and consists mainly of specific positive mechanical work $e_{\text {mech }}^{+}$. At a speed of $\bar{v}=2.3 \mathrm{~m} / \mathrm{s}$ the specific positive mechanical work contributes $81.5 \%$ of the specific cost of transport. This implies that the positive mechanical work $e_{\text {mech }}^{+}$is not negligible and the specific static work $e_{\text {stat }}$, commonly used in optimal control approaches $[18,32]$, is not a suitable objective function if energy efficiency is considered. The energy input by positive specific mechanical work $e_{\text {mech }}^{+}$is primarily dissipated by the specific negative mechanical work $e_{\text {mech }}^{-}$. At a speed of $\bar{v}=2.3 \mathrm{~m} / \mathrm{s} 88.7 \%$ of the specific positive mechanical work is consumed by the specific negative mechanical work. This implies that the optimization minimizes the specific impact loss.

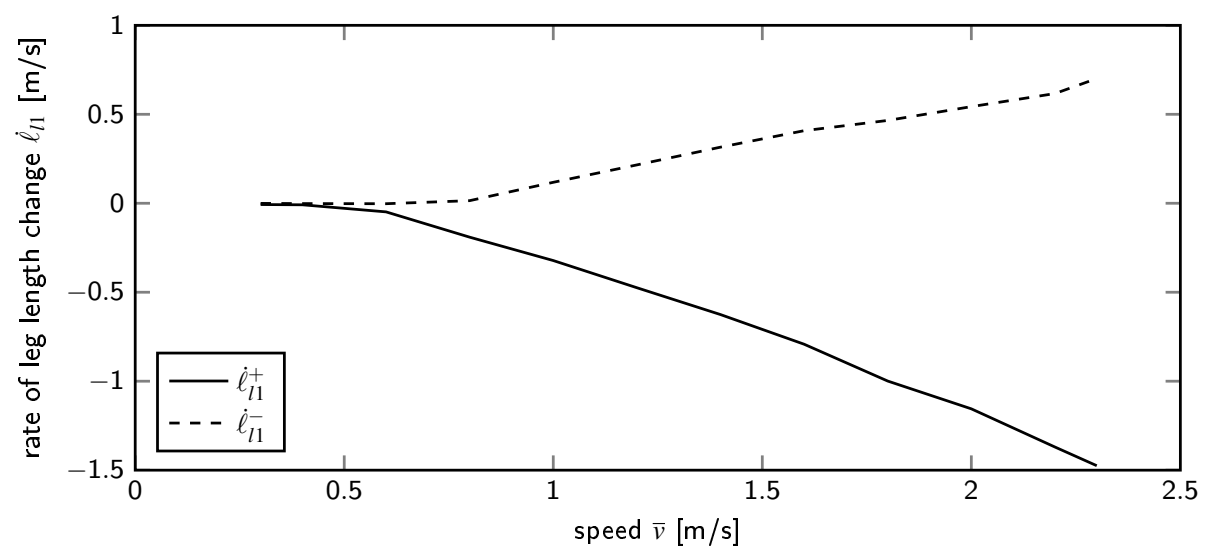

Fig. 9 Rate of the stance leg's effective length change after $\dot{\ell}_{l 1}^{+}$and before $\dot{\ell}_{l 1}^{-}$the impact

Figure 9 shows the rate of the stance leg's effective length change after $\dot{\ell}_{l 1}^{+}$and before $\dot{\ell}_{l 1}^{-}$the impact. By lengthening the stance leg before the impact $\dot{\ell}_{l 1}^{-}>0$, the robot's center of mass motion is diverted from a circular motion about the stance leg foot 1 to a more horizontal path. Therefore, the absolute value of the robot's vertical center of mass velocity $\dot{\mathbf{r}}_{C O M}$ and the potential impact loss is reduced. By shortening the stance leg after the impact $\dot{\ell}_{l 1}^{-}<0$, the knee flexion of the stance leg acts as a shock absorber. The flexion of the knee joint increases the lever arm of the acting impact force. This allows for the transfer of the translational kinetic energy to rotational kinetic energy of the thigh and shank rotation instead of its dissipation in the impact loss. In order to break against the acceleration of the thigh and shank rotation specific negative mechanical work is needed.

The measure of enlarging the lever arm of the impact force, transferring the translational kinetic energy to rotational kinetic energy and breaking the rotation during the step in order to reduce the impact loss is also used in the hip joint between trunk and thigh and is one reason for the increase of the trunk inclination angle with increasing speed (cf. Fig. 7). This means that a strategy to reduce the specific cost of transport has to decrease the specific negative mechanical work by finding another measure to reduce the specific impact loss.

In order to find an appropriate measure to reduce the impact loss, its origin is investigated with the simplest walking model consisting of an inverted mathematical pendulum as depicted in Fig. 10. During the single support phase the hip, containing the total mass, moves on a circular path around the foot of the massless stance leg. The double support 


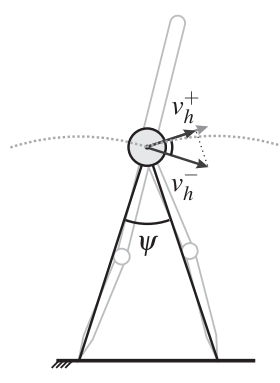

Fig. 10 Explanation of the impact loss by an inverted mathematical pendulum as simple model for the double support phase

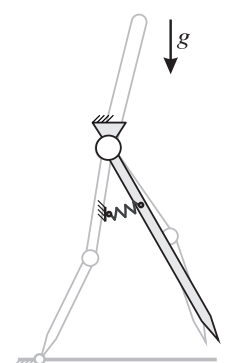

Fig. 11 Explanation of the double step frequency by a physical pendulum as simple model for the single support phase

phase corresponds to the transition from one circular path to the next meaning that the impact is described by a projection of the hip velocity $v_{h}^{+}=v_{h}^{-} \cos \psi$ into the new tangential direction. Therefore, the specific impact loss of the inverted pendulum

$$
e_{\text {imp } p_{i p}}=\frac{\left(v_{h}^{-}\right)^{2}}{2 g \ell_{l}} \sin \psi \cos \frac{1}{2} \psi
$$

can be determined analytically and is valid in the stiction domain of the leg opening angle $\psi \in\left[0,2 \arctan \left(\mu_{0}\right)\right]$. Equation (80) directly suggests an opportunity to reduce the specific impact loss. By reducing the robot's leg opening angle $\psi$ and therewith the step length $r_{2_{x}}\left(\theta^{-}\right)$the specific impact loss of the inverted pendulum $e_{i m p_{i p}}$ decreases and finally disappears. This raises the question why the optimization does not converge to this solution. Since the robot has to walk at a specific speed, a decrease of step length means an increase of step frequency.

In order to evaluate the double step frequency ${ }^{10}$ of the optimized motion, the swing leg, which is the body with the highest acceleration in the single support phase, is modeled as physical pendulum (cf. Fig. 11). By linearizing the equation of motion about the lower rest position, the eigenfrequency

$$
f_{S M_{0}}=\frac{1}{2 \pi} \sqrt{\frac{m_{l} g r_{l}+k_{l_{l} l}}{J_{l}+m_{l} r_{l}^{2}}}
$$

of the swing leg model can be determined. The numerical values of the physical pendulum's parameters $\left(r_{l}, m_{l}, J_{l}\right)$ are determined by averaging the values of the swing leg consisting of thigh and shank during one step. In the entire speed range the double step frequency $f_{D S}$ of the optimized motion of the robot is significantly above the eigenfrequency $f_{S M_{0}}$ of the swing leg model (cf. Fig. 12). At a speed of $\bar{v}=2.3 \mathrm{~m} / \mathrm{s}$ the double step frequency equals 2.56 times the eigenfrequency of the swing leg model. This means the optimized motion is already far above resonance operation and to diverge further by making smaller steps is not energetically efficient. However, the swing leg model also presents an opportunity to increase the eigenfrequency of the swing leg model via introduction of an elastic coupling of the swing leg with the stiffness $k_{l \_l}$ (cf. Eq. (81)).

In the remainder of this section a robot with linear elastic coupling between the shanks $\left(l s \_l s\right)$ is investigated (cf. Fig. 2(b)). The robot's motion $(\boldsymbol{\alpha})$ for each speed as well as

\footnotetext{
10 One double step corresponds to one cycle of walking and consists of one step with the right leg and one step with the left leg.
} 


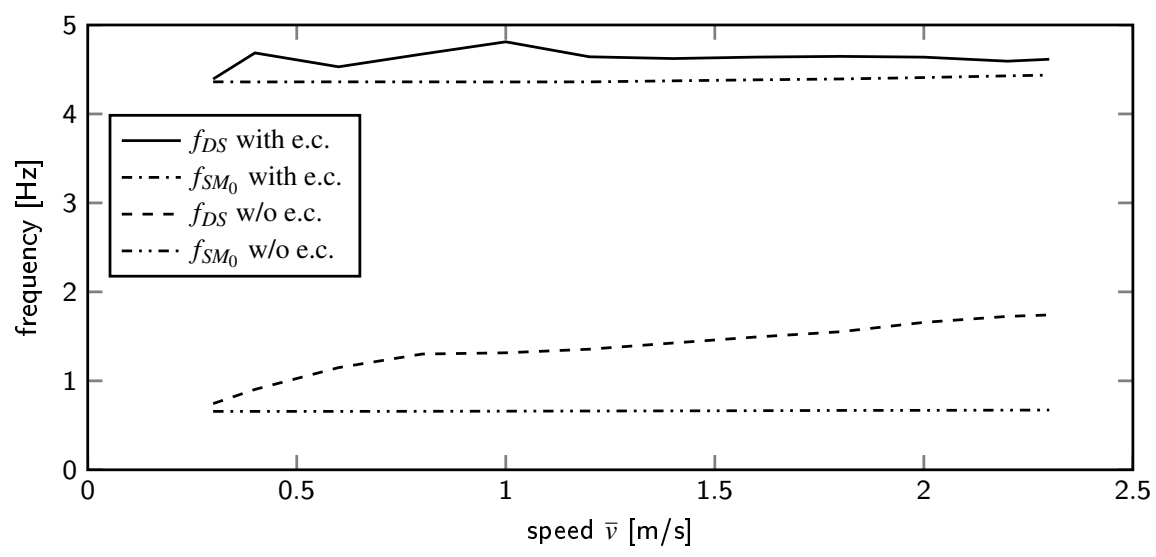

Fig. 12 Comparison of the double step frequency $f_{D S}$ of the optimized motion with the eigenfrequency $f_{S M_{0}}$ of the swing leg model for the robot with and without elastic coupling

the elastic coupling $(\boldsymbol{\beta})$ are optimized by the process described in Sec. 3. This results in the movement speed independent stiffness $k_{l s_{-} l s}=2250 \mathrm{Nm} / \mathrm{rad}$ and the transmission ratio $i_{l s_{-} l s}=0.364$ as well as the associated motions.

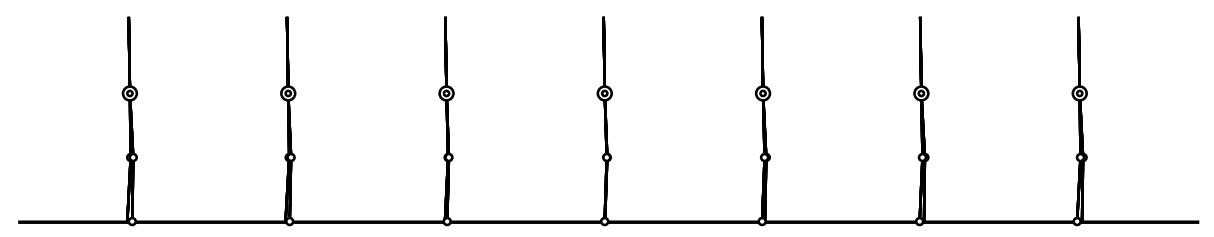

(a) $\bar{v}=0.3 \mathrm{~m} / \mathrm{s}$

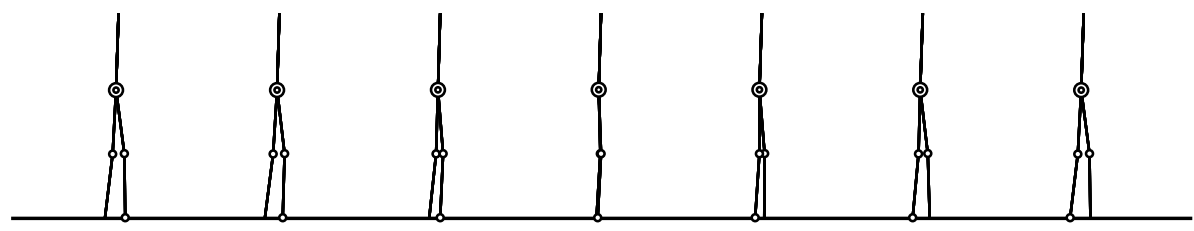

(b) $\bar{v}=1.3 \mathrm{~m} / \mathrm{s}$

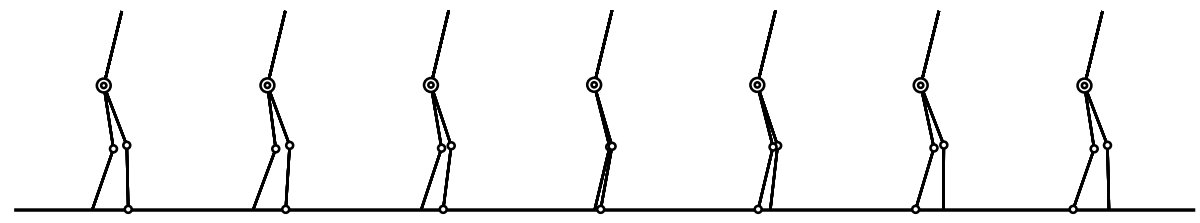

(c) $\bar{v}=2.3 \mathrm{~m} / \mathrm{s}$

Fig. 13 Comparison of the robot's motion with elastic coupling of the shanks between the legs $\left(l s_{-} l s\right)$ at three different speeds $\bar{v}$ at seven equidistant instances of time 
Figure 13 displays the resulting motions for the lowest $(\bar{v}=0.3 \mathrm{~m} / \mathrm{s})$, medium $(\bar{v}=$ $1.3 \mathrm{~m} / \mathrm{s})$ and highest $(\bar{v}=2.3 \mathrm{~m} / \mathrm{s})$ considered speed at seven equidistant instances of time. Through comparison of the kinematics on position level of the motion of the robot without elastic coupling (Fig. 7) with the motion of the robot with elastic coupling (Fig. 13) three patterns emerge.

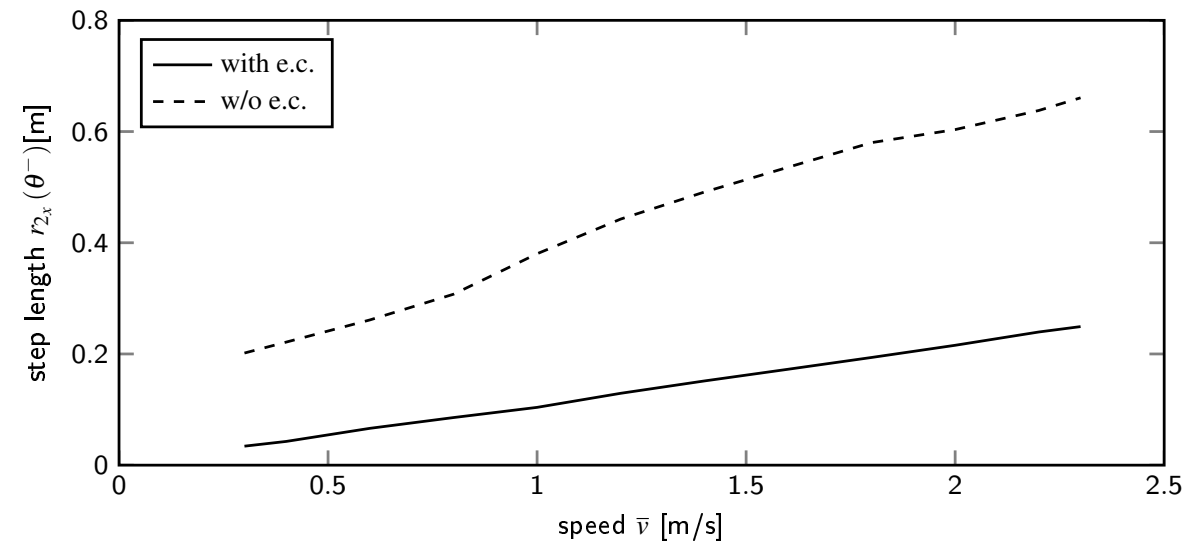

Fig. 14 Step length $r_{2 x}\left(\theta^{-}\right)$of the robot with and without elastic coupling

First, the step length $r_{2_{x}}\left(\theta^{-}\right)$of the robot with elastic coupling is considerably smaller than the step length of the robot without elastic coupling as depicted in Fig. 14. At the speed of $\bar{v}=2.3 \mathrm{~m} / \mathrm{s}$ the step length of the robot is reduced from $0.661 \mathrm{~m}$ to $0.249 \mathrm{~m}$ which corresponds to a reduction of $62.3 \%$. This indicates the expected phenomenon of reduction of impact loss. Furthermore, the step length of the robot with elastic coupling is almost a linear function of speed, which indicates a motion with constant double step frequency.

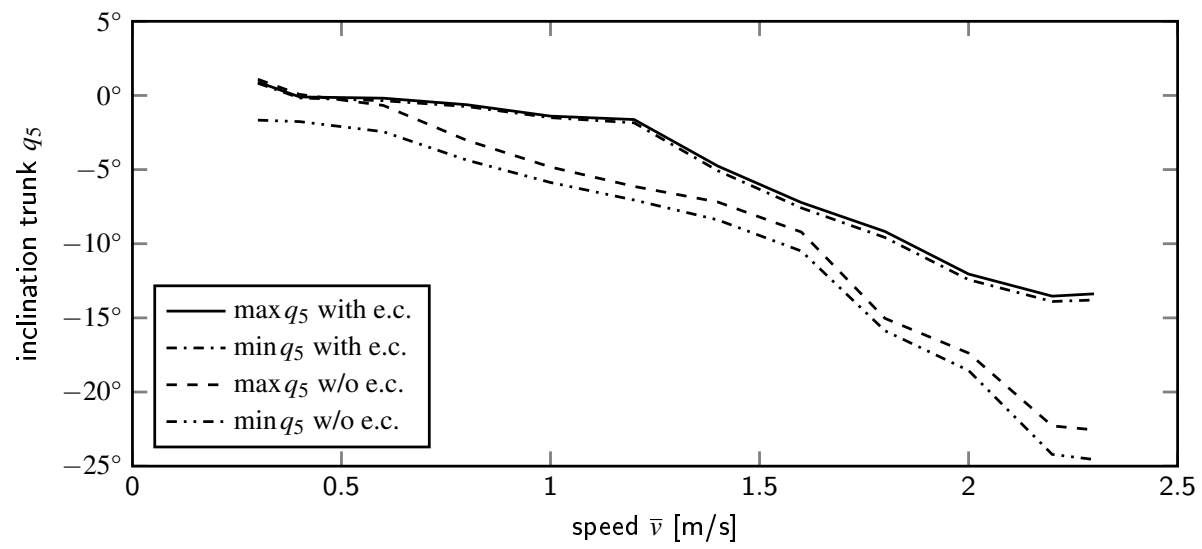

Fig. 15 Trunk inclination angle $q_{5}$ of the robot with and without elastic coupling 
Second, the trunk inclination angle $q_{5}$ of the robot with elastic coupling is much smaller compared to the robot without elastic coupling as depicted in Fig. 15. At the speeds $\bar{v}=$ $0.3 \mathrm{~m} / \mathrm{s}$ and $\bar{v}=2.3 \mathrm{~m} / \mathrm{s}$, the maximum trunk inclination angle of the robot is reduced from $1.09^{\circ}$ and $24.5^{\circ}$ to $0.68^{\circ}$ and $13.8^{\circ}$ respectively. This corresponds to a reduction of $38.1 \%$ and $43.8 \%$ due to the elastic coupling. This indicates that the impact loss is reduced elsewhere and it is not necessary to maximize the impact force's lever arm. This hypothesis is further confirmed by the reduced trunk inclination angle sway at $\bar{v}=2.3 \mathrm{~m} / \mathrm{s}$ from $2.00^{\circ}$ to $0.415^{\circ}$ which corresponds to a reduction of $79.2 \%$; the trunk inclination angle of the robot with elastic coupling remains almost constant.

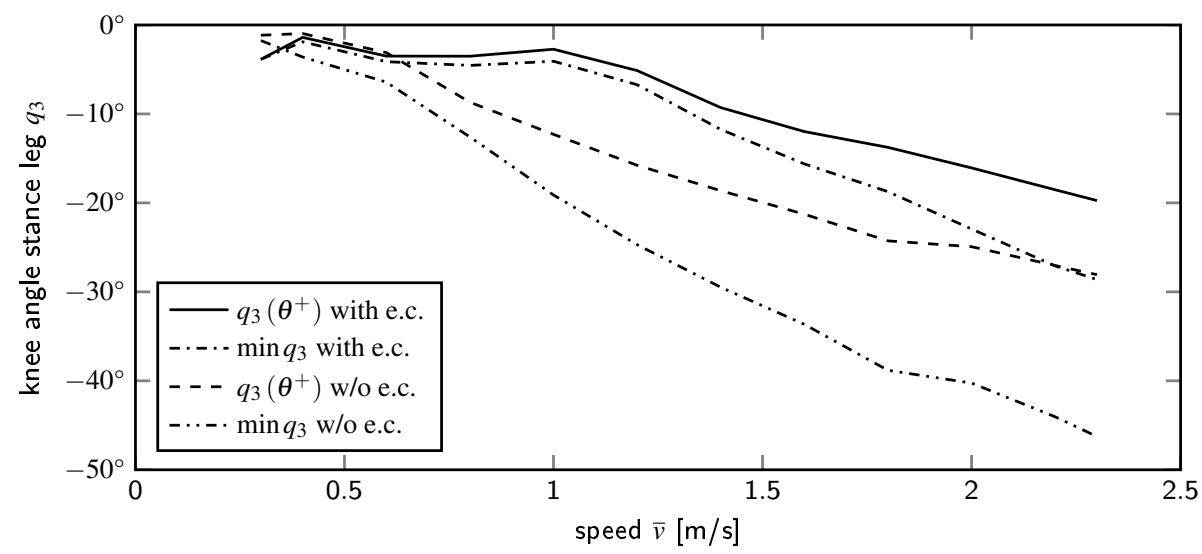

Fig. 16 Stance leg knee flexion angle $q_{3}$ of the robot with and without elastic coupling

Third, the stance leg knee flexion angle after the impact $q_{3}\left(\theta^{+}\right)$and its regime of braking $q_{3}\left(\theta^{+}\right)-\min q_{3}$ of the robot with elastic coupling is smaller than of the robot without elastic coupling as depicted in Fig. 16. At the speed of $\bar{v}=2.3 \mathrm{~m} / \mathrm{s}$ the regime of braking is reduced from $18.2^{\circ}$ to $8.88^{\circ}$ which corresponds to a reduction of $51.2 \%$. This also indicates that the impact loss is reduced elsewhere and the leg needs to act as shock absorber less strongly.

Figure 12 depicts the double step frequency $f_{D S}$ of the optimized motion and the eigenfrequency $f_{S M_{0}}$ of the swing leg model of the robot with and without elastic coupling. The elastic coupling of the robot is assumed to act directly at the hip with the stiffness $k_{l \_l}=k_{l s \_l s}$. By the use of the elastic coupling the eigenfrequency of the swing leg model and thus the natural frequency of the robot is increased. At the speed of $\bar{v}=2.3 \mathrm{~m} / \mathrm{s}$ the eigenfrequency of the swing leg model increases from $0.672 \mathrm{~Hz}$ to $4.44 \mathrm{~Hz}$. Over the total speed range the double step frequency of the optimized motion is almost constant within a span of $9.09 \%$ and a maximal deviation from the eigenfrequency of the swing leg model of $10.4 \%$. Therefore, we conclude that the robot moves in resonance.

Figure 17 illustrates the different specific energy losses of the robot with and without elastic coupling. The sum of the specific negative mechanical work $e_{\text {mech }}^{-}$and the specific impact loss $e_{i m p}$ has to be compensated by specific positive mechanical work $e_{\text {mech }}^{+}$and therefore determines the specific cost of transport $c_{T}$. Introducing the elastic coupling mainly reduces the specific negative mechanical work $e_{m e c h}^{-}$. This confirms the aforementioned hypothesis. Moreover, the specific impact loss $e_{i m p}$ is reduced. In the lower to medium speed 


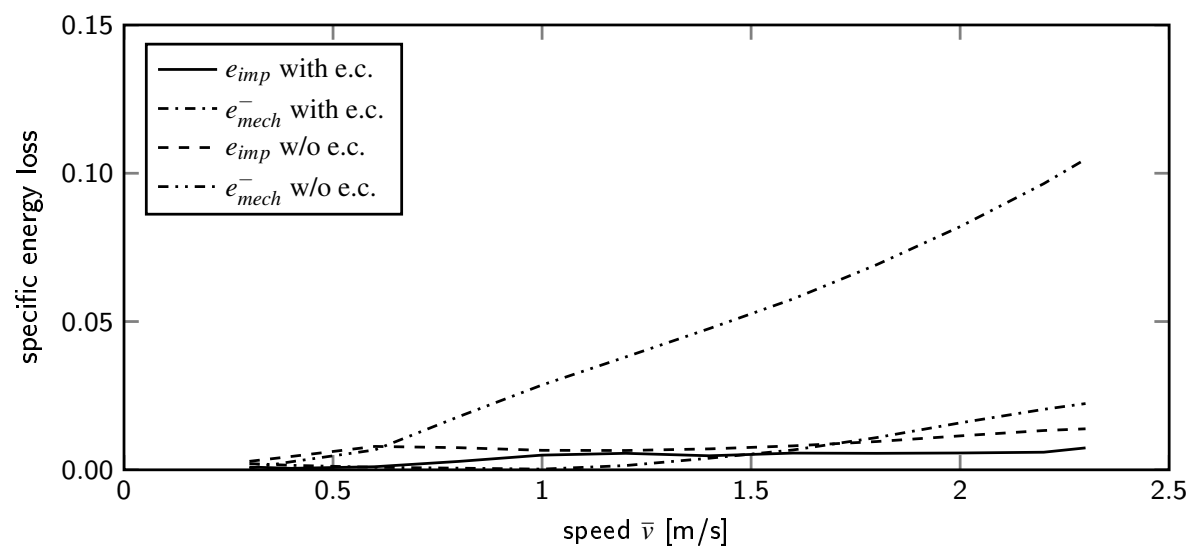

Fig. 17 Comparison of the specific energy loss of the robot with and without elastic coupling

range $\bar{v}=0.3-1.3 \mathrm{~m} / \mathrm{s}$ the specific negative mechanical work almost disappears. It increases in the medium to higher speed range $\bar{v}=1.3-2.3 \mathrm{~m} / \mathrm{s}$, because the shock absorbing function and therewith the breaking function of the knee increase with a growing knee flexion angle (cf. Fig. 16) .

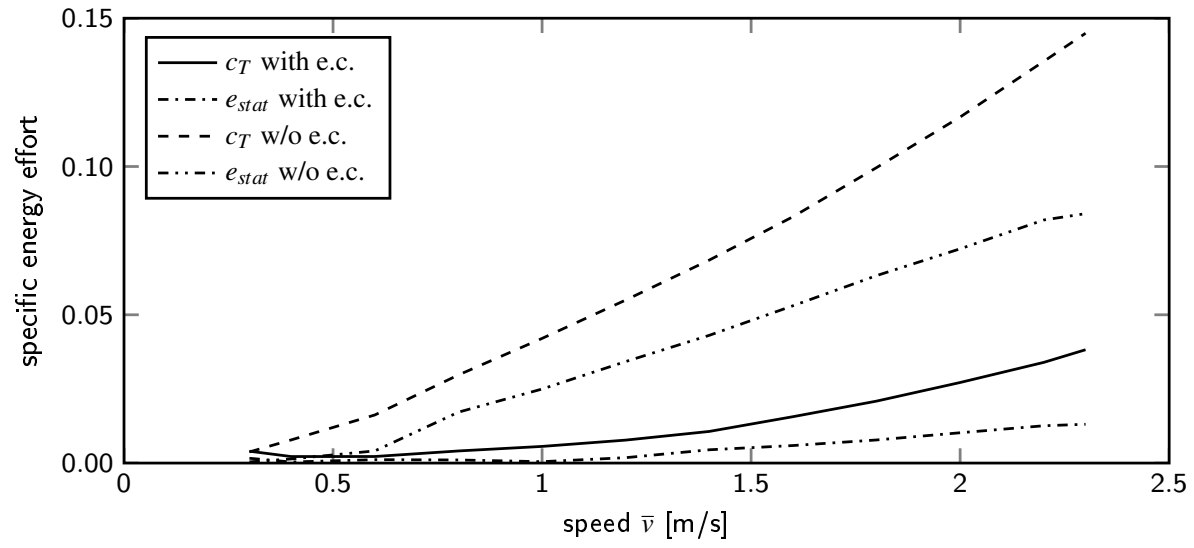

Fig. 18 Comparison of the robot's specific energy effort with and without elastic coupling

Figure 18 shows the specific energy efforts of the robot with and without elastic coupling. By introduction of the elastic coupling the specific cost of transport $c_{T}$ is reduced in the entire speed range. The mean specific cost of transport $\bar{c}_{T}$ for the speed range $\bar{v}=$ $0.3-2.3 \mathrm{~m} / \mathrm{s}$ is reduced from $\bar{c}_{T}=0.0654$ to $\bar{c}_{T}=0.0130$ which is a saving of $80.1 \%$. The mean specific static energy $\bar{e}_{\text {stat }}$, which corresponds to the energy dissipation in form of heat in the actuator's armature resistor, is reduced from $\bar{e}_{\text {stat }}=0.0395$ to $\bar{e}_{\text {stat }}=0.00458$ which is a saving of $88.4 \%$. The elastic coupling does not only reduce the specific cost of transport significantly, it also allows for a downsizing of the actuators, which might lead to a further reduction of specific cost of transport since this changes $c_{\text {stat }}$. 
Summing up, the elastic coupling increases the natural frequency of the swing leg motion and therefore allows for a resonance operation with small step length which reduces the impact loss and therewith supersedes the shock absorber mode of the knee flexion demanding for high specific negative mechanical work and thus reduces the specific cost of transport significantly.

\subsection{Influence of the elastic coupling's topology}

While the reduction of the specific cost of transport was illustrated by the example of the linear elastic coupling of the shanks (lin. $l s_{-} l s$ ) in the previous section, in this section the influence of the elastic coupling's topology is investigated. In order to evaluate the efficiency of a single elementary elastic coupling in a combination of elastic couplings, those with similar effect are compared. As illustrated in Fig. 15 the trunk inclination angle of the robot with linear elastic coupling of the shanks is almost constant during one step. This means the direct elastic coupling of the shanks $\left(l s_{-} l s\right)$ between the legs and the indirect elastic coupling of the shanks via elastic coupling of trunk and shank $\left(t_{-} l s\right)$ within each leg have an almost identical effect (cf. Fig. 2(b)), provided the resting angle $\varphi_{t_{-} l s_{0}}$ of the elastic coupling of trunk and shank is chosen properly. Analogously, the elastic couplings of the thighs and of thigh and shank between the legs and within each leg can be considered as direct and indirect elastic couplings with similar functions. In the following, the elastic couplings between the legs and within each leg depicted in Fig. 2 with characteristics described by Eq. (25) are evaluated regarding their efficiency by means of the specific cost of transport $c_{T}$ and compared to a robot without elastic coupling. In each case the motion as well as the elastic coupling are optimized with the process described in Sec. 3. The elastic couplings' parameters $\boldsymbol{\beta}$ and their resulting mean specific cost of transport $\bar{c}_{T}$ as well as their relative saving $\Delta \bar{c}_{T} / \bar{c}_{T \_0}$ are collected in Table 3 .

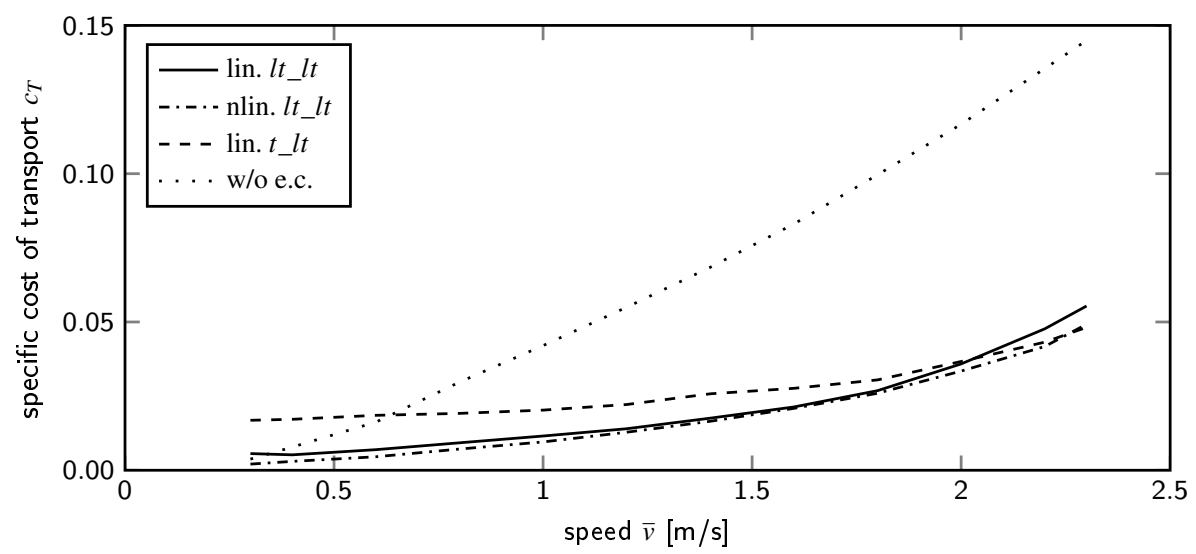

Fig. 19 Comparison of the specific cost of transport $c_{T}$ of different elastic couplings of the thighs between the legs $\left(l t \_l t\right)$ and within each leg $\left(t \_l t\right)$

Figure 19 depicts the specific cost of transport $c_{T}$ of the robot with elastic coupling of the thighs between the legs (lt_lt) and within each leg $\left(t_{-} l t\right)$ as well as without elastic coupling (w/o e.c.). Because of a movement speed dependent mean trunk inclination angle 
(cf. Fig 15), the movement speed independent resting angle $\varphi_{t_{-} l t_{0}}=3.49 \mathrm{rad}$ of the thighs' elastic coupling within each leg $\left(t \_l t\right)$ has to be a compromise. For low speeds $(\bar{v}=0.3-$ $0.6 \mathrm{~m} / \mathrm{s}$ ) this results in a specific cost of transport of the robot with linear elastic coupling within each leg (lin. $t_{-} l s$ ) which is worse than the one of the robot without elastic coupling (w/o e.c.). For medium and higher speeds $(\bar{v}=0.6-2.0 \mathrm{~m} / \mathrm{s})$ the specific cost of transport of the robot with linear elastic coupling within each leg (lin. $t_{-} l t$ ) is better than the version without elastic couplings (w/o e.c.). Only for the highest speeds $(\bar{v}=2.0-2.3 \mathrm{~m} / \mathrm{s})$ it is better than the version with linear elastic coupling between the legs (lin. $l t \_l t$ ) because the elastic coupling within each leg can be used to suspend the torque of gravity caused by the trunk inclination. However, the mean specific cost of transport $\bar{c}_{T}=0.02617$ of the robot with linear elastic coupling within each leg (lin. $t$ l $l$ ) is far above the mean specific cost of transport $\bar{c}_{T}=0.01963$ of the robot with linear elastic coupling between the legs (lin. lt_lt) which can be further reduced $\bar{c}_{T}=0.01756$ by a nonlinear elastic coupling between the legs (nlin. $l t \_l t$ ) which constitutes the optimal elastic coupling of the thighs.

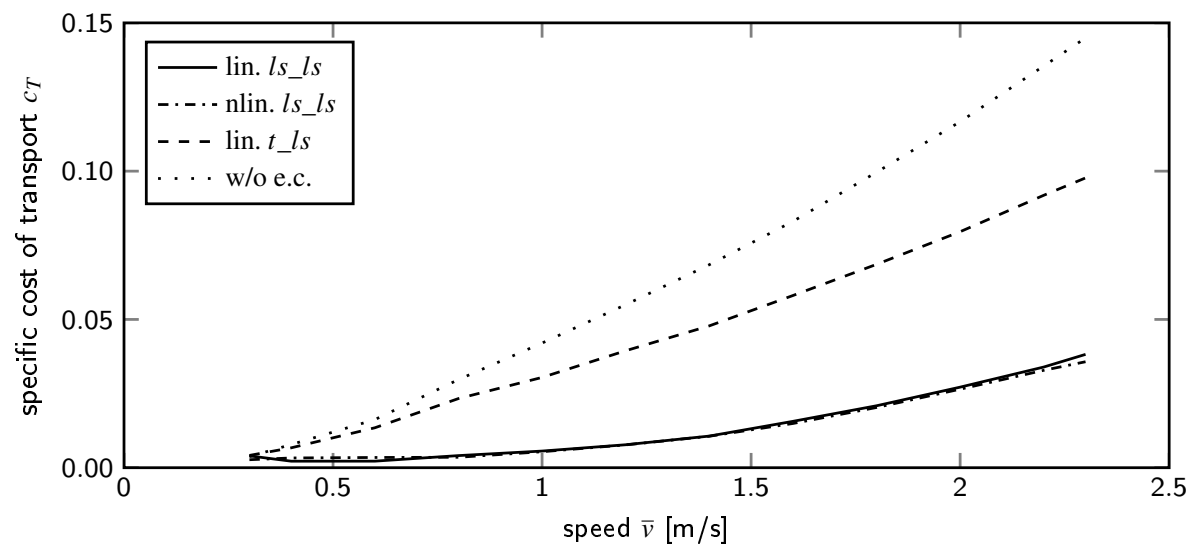

Fig. 20 Comparison of the specific cost of transport $c_{T}$ of different elastic couplings of the shank between the legs $\left(l s \_l s\right)$ and within each leg $\left(t_{-} l s\right)$

Figure 20 displays the specific cost of transport $c_{T}$ of the robot with elastic coupling of the shanks between the legs $\left(l s_{-} l s\right)$ and within each leg $\left(t_{-} l s\right)$ as well as without elastic coupling (w/o e.c.). As already explained in the last paragraph, the resting angle $\varphi_{t} l s_{0}=$ 3.06 rad and thus the shanks' elastic coupling within each leg $\left(t_{-} l s\right)$ has to be a compromise because of the movement speed dependent trunk inclination angle. The mean specific cost of transport $\bar{c}_{T}=0.04593$ of the robot with linear elastic coupling between trunk and legs (lin. $t_{-} l s$ ) is far above the mean specific cost of transport $\bar{c}_{T}=0.01302$ of the robot with linear elastic coupling between the legs (lin. $l s \_l s$ ) which can be reduced only marginally to $\bar{c}_{T}=0.01287$ by a nonlinear elastic coupling between the legs (nlin. $l s_{-} l s$ ). Since the degree of nonlinearity $v_{l s}$ ls $=1.10$ is rather low and the effort for the nonlinear design outweighs its advantages, the linear elastic coupling between the legs (lin. $l s \_l s$ ) is considered as optimal elastic coupling of the shanks.

Figure 21 illustrates the specific cost of transport $c_{T}$ of the robot with elastic coupling of thigh and shank between the legs $\left(l t 1 \_l s 2\right)$ and within each leg $\left(l t \_l s\right)$ together with the robot without elastic coupling (w/o e.c.). Because of a speed dependent mean knee flexion angle and different torque directions in the stance leg knee joint and the swing leg knee joint at the 


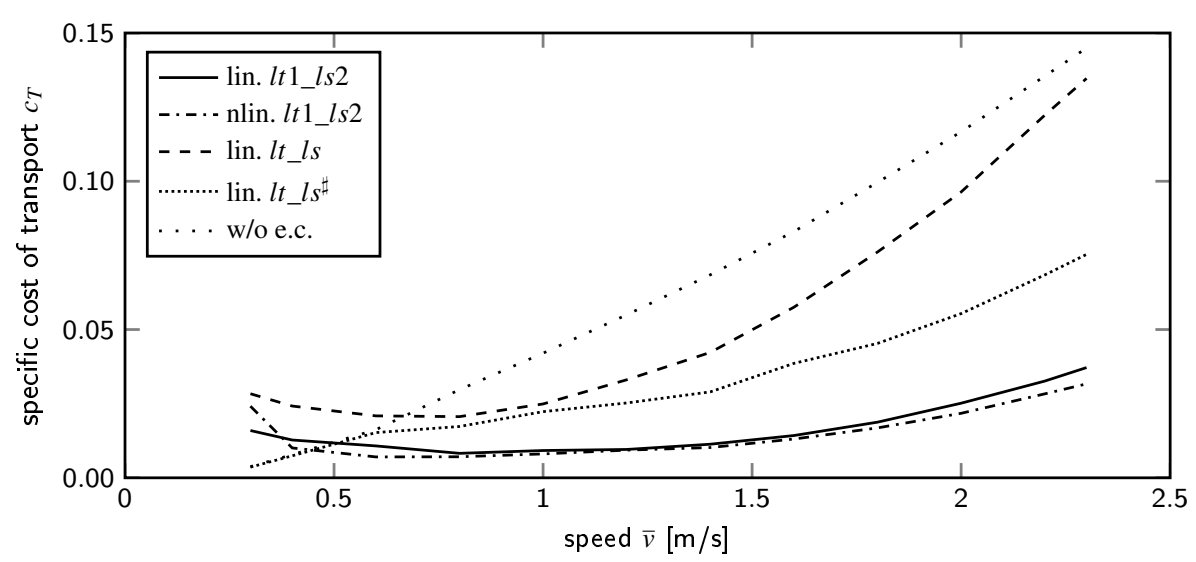

Fig. 21 Comparison of the specific cost of transport $c_{T}$ of different elastic couplings of thigh and shank between the legs $\left(l t 1 \_l s 2\right)$ and within each leg $\left(l t \_l s\right)$

same knee angle, it is hard to find a good compromise for the resting angle $\varphi_{t \_l} l s_{0}=3.06 \mathrm{rad}$ and thus the thigh and shank's elastic coupling within each leg $\left(l t \_l s\right)$. Hence, the curve of the specific cost of transport of the robot with linear elastic coupling within each leg (lin. $l t \_l s$ ) is above the curve of the robot without elastic couplings (w/o e.c.) for low speeds $\bar{v}=0.3-0.7 \mathrm{~m} / \mathrm{s}$ and below it but far above the curve of the robot with linear elastic coupling between the legs (lin. $l t 1 \_l s 2$ ) for medium and high speeds $\bar{v}=0.7-2.3 \mathrm{~m} / \mathrm{s}$. In order to avoid this issue, a switchable linear elastic coupling between thigh and shank (lin. $\left.l t \_l s^{\sharp}\right)$ with a clutch is considered. The elastic coupling is only active in the stance leg; the clutch closes at touchdown of the foot with a relaxed elastic coupling and opens at takeoff. The energy saved in the elastic coupling is lost when the clutch opens. The mean specific cost of transport $\bar{c}_{T}=0.05201$ of the robot with linear elastic coupling within each leg (lin. $l t \_l s$ ) is lowered by the design with switchable elastic coupling to $\bar{c}_{T}=0.03242$, however it is still above the mean specific cost of transport $\bar{c}_{T}=0.01527$ of the robot with linear elastic coupling between the legs (lin. $l t 1_{-} l s 2$ ). It is further improved to $\bar{c}_{T}=0.01318$ by a nonlinear elastic coupling between the legs (nlin. $\left.l t 1 \_l s 2\right)$ which constitutes the optimal elastic coupling of thigh and shank.

Because of their similar effect a combination of the elementary elastic couplings within each leg and their corresponding elementary elastic couplings between the legs does not show improvements in the specific cost of transport. In order to find the optimal elastic coupling of the robot, the different elementary elastic couplings between the legs are compared and combined if necessary.

Figure 22 shows the specific cost of transport $c_{T}$ of each robot with one elementary elastic coupling between the legs. The mean specific cost of transport $\bar{c}_{T}=0.01756$ of the robot with elastic coupling of the thighs (nlin. $l t \_l$ ) is significantly worse than the mean specific cost of transport $\bar{c}_{T}=0.01302$ of the robot with elastic coupling of the shanks (lin. $\left.l s_{-} l s\right)$ and of the mean specific cost of transport $\bar{c}_{T}=0.01318$ of the robot with elastic coupling of thigh and shank (nlin. $l t 1 \_l s 2$ ) which lie close together. From lower to medium speeds $(\bar{v}=0.3-1.4 \mathrm{~m} / \mathrm{s})$ the elastic coupling of the shanks is advantageous, from medium to higher speeds $(\bar{v}=1.4-2.3 \mathrm{~m} / \mathrm{s})$ the elastic coupling of thigh and shank performs better with regard to the specific cost of transport. 


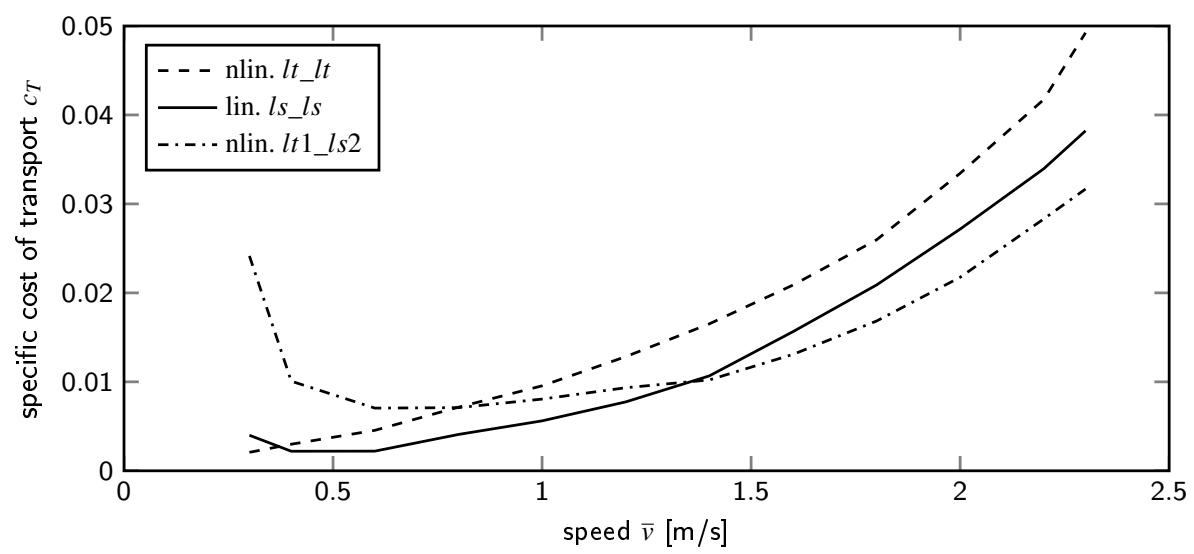

Fig. 22 Comparison of the specific cost of transport $c_{T}$ of the optimal elastic couplings of the thighs, the shanks and of thigh and shank between the legs

Combinations of the elastic couplings of the thighs, of the shanks and of thigh and shank show no further improvement of the specific cost of transport. In order to analyze this issue the joint torques $\mathbf{T}_{e c}$ of the linear elementary elastic couplings between the legs are represented with respect to the configuration $\mathbf{q}=\left[q_{1}, q_{2}, q_{3}, q_{4}, q_{5}\right]^{T}$ :

$$
\begin{aligned}
& \mathbf{T}_{l t_{-} l t}=-k_{l t_{-} l t}\left[\begin{array}{c}
-\left(q_{2}-q_{1}\right) \\
\left(q_{2}-q_{1}\right) \\
0 \\
0 \\
0
\end{array}\right]_{\mathbf{q}} \\
& \mathbf{T}_{l s_{-} l s}=-k_{l s_{-} l s}\left[\begin{array}{c}
-\left(q_{2}-q_{1}+i_{l s_{-} l s}\left(q_{4}-q_{3}\right)\right) \\
\left(q_{2}-q_{1}+i_{l s_{-} l s}\left(q_{4}-q_{3}\right)\right) \\
-i_{l s_{-} l s}\left(q_{2}-q_{1}+i_{l s_{-} l s}\left(q_{4}-q_{3}\right)\right) \\
i_{l s_{-} l s}\left(q_{2}-q_{1}+i_{l s_{-} l s}\left(q_{4}-q_{3}\right)\right) \\
0
\end{array}\right]_{\mathbf{q}}, \\
& \mathbf{T}_{l t 1 \_l s 2}=-2 k_{l t 1 \_l s 2}\left[\begin{array}{c}
-\left(q_{2}-q_{1}+\frac{1}{2} i_{l t 1 \_l s 2}\left(q_{4}-q_{3}\right)\right) \\
\left(q_{2}-q_{1}+\frac{1}{2} i_{l t 1 \_l s 2}\left(q_{4}-q_{3}\right)\right) \\
-\frac{1}{2} i_{l t 1 \_l s 2}\left(q_{2}-q_{1}+i_{l t 1 \_l s 2}\left(\frac{\varphi_{l t 1 \_s 2_{0}}}{\left.i_{l t 1 \_l s 2}-q_{3}\right)}\right)\right) \\
\frac{1}{2} i_{l t 1 \_l s 2}\left(q_{2}-q_{1}+i_{l t 1 \_l s 2}\left(q_{4}-\frac{\varphi_{l t \_s 2_{0}}}{i_{l t 1 \_l s 2}}\right)\right) \\
0
\end{array}\right]_{\mathbf{q}}
\end{aligned}
$$

By setting the transmission ratio of the knee joints $i_{l s_{-} l s}$ and $i_{l t 1 \_l s 2}$ to zero, the joint torque $\mathbf{T}_{l t l t}$ of the elastic coupling of the thighs (cf. Eq. (82)) can be represented by the joint torque $\mathbf{T}_{l s \_l s}$ of the elastic coupling of the shanks (cf. Eq. (83)) and the joint torque $\mathbf{T}_{l t 1 \_l s 2}$ of the elastic coupling of thigh and shank (cf. Eq. (84)). Therefore, the elastic coupling of the thighs is already implicitly included in the other two and contributes no additional function. The joint torque $\mathbf{T}_{l s \_l s}$ of the elastic coupling of the shanks and the joint torque $\mathbf{T}_{l t 1 \_l s 2}$ of the elastic coupling of thigh and shank differ only in the torques with respect to the knee joints in the value of the respective knee joint angle $\left(q_{3}\right.$ and $\left.q_{4}\right)$. Since the difference in the hip joint angles $q_{2}-q_{1}$ is not reduced and much bigger than the difference of the knee joint 
Table 3 Comparison of different elastic couplings between the legs and within each leg by parameters $\boldsymbol{\beta}$ and resulting mean specific cost of transport $\bar{c}_{T}$ with relative saving $\Delta \bar{c}_{T} / \bar{c}_{T_{-} 0}$

\begin{tabular}{|c|c|c|c|c|c|c|c|c|}
\hline & \multicolumn{2}{|c|}{ elastic coupling } & $\varphi_{0}[\mathrm{rad}]$ & $k\left[\frac{\mathrm{Nm}}{\mathrm{rad}}\right]$ & $v$ & $i$ & $\bar{c}_{T}$ & $\Delta \bar{c}_{T} / \bar{c}_{T \_0}$ \\
\hline & \multicolumn{5}{|l|}{ w/o } & & \multicolumn{2}{|l|}{0.06540} \\
\hline \multirow{4}{*}{ 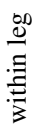 } & $t \_l t$ & lin. & 3.49 & 1090 & 1 & \multirow{4}{*}{0.800} & 0.02617 & $60.0 \%$ \\
\hline & $t \_l s$ & lin. & 3.06 & 2250 & 1 & & 0.04593 & $29.8 \%$ \\
\hline & $\overline{l t} \_l s$ & lin. & -0.475 & 880 & 1 & & 0.05201 & $20.5 \%$ \\
\hline & $l t \_l s^{\sharp}$ & lin. & N/A & 374 & 1 & & 0.03242 & $50.4 \%$ \\
\hline \multirow{6}{*}{ 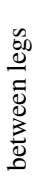 } & lt_lt & lin. & 0 & 785 & 1 & & 0.01963 & $70.0 \%$ \\
\hline & $l t \_l t$ & nlin. & 0 & 2490 & 1.93 & & 0.01756 & $73.2 \%$ \\
\hline & $l s \_l s$ & lin. & 0 & 2250 & 1 & 0.364 & 0.01302 & $80.1 \%$ \\
\hline & $l s \_l s$ & nlin. & 0 & 2740 & 1.10 & 0.345 & 0.01287 & $80.3 \%$ \\
\hline & $l t 1 \_l s 2$ & lin. & -0.183 & 1040 & 1 & 0.678 & 0.01527 & $76.7 \%$ \\
\hline & $l t 1 \_l s 2$ & nlin. & -0.198 & 2400 & 1.36 & 0.818 & 0.01318 & $79.9 \%$ \\
\hline
\end{tabular}

angles $q_{4}-q_{3}$, the elastic coupling of the shanks and the elastic coupling of thigh and shank have a similar function. A combination of the elementary elastic couplings of the shanks and of thigh and shank only influences the curve of the specific cost of transport but not its mean value.

With regard to the implementation of the elastic coupling in hardware a simple design is preferred. Hence the linear elementary elastic coupling of the shanks (lin. $l s \_l s$ ) is considered as the optimal elastic coupling. By using only one elastic coupling in form of a simple mechanical spring, the mean specific cost of transport $\bar{c}_{T}$ for the speed range $\bar{v}=0.3-2.3 \mathrm{~m} / \mathrm{s}$ is reduced from $\bar{c}_{T}=0.06540$ to $\bar{c}_{T}=0.01302$ which is a reduction of $80.1 \%$. In the remainder of this paper the robot equipped with this elastic coupling is investigated further.

\subsection{Influence of viscous joint damping}

In the previous sections the academic case of a robot without joint friction was considered. In this section the influence of viscous joint damping on the reduction of specific cost of transport by elastic couplings is investigated. As shown in Sec. 4.1 the reduction is mainly caused by an increase of the resonance frequency of the swing leg motion and thus by increasing the joint angular velocity. The joint torque caused by viscous joint damping counteracts the motion and is proportional to the angular velocity. Hence, a decrease of the reduction of specific cost of transport by elastic couplings is anticipated. In order to quantify the influence of viscous joint damping the mechanical model depicted in Fig. 1 is enhanced by linear rotational joint dampers with damping coefficient $d_{J}=8 \mathrm{Nm} \mathrm{s} / \mathrm{rad}$ corresponding to the value of the assumed reduced drive train (cf. Sec. 2.2). The motion as well as the elastic coupling of the robot are optimized if applicable.

Figure 23 displays the absolute saving of specific cost of transport $\Delta c_{T}$ by the elastic coupling with and without viscous joint damping. Surprisingly the absolute saving in specific cost of transport by the elastic coupling depends only weakly on the joint damping coefficient.

Figure 24 illustrates the relative saving of the specific cost of transport $\Delta c_{T} / c_{T}$ o by the elastic coupling with and without viscous joint damping. Since the specific cost of transport $c_{T_{-} 0}$ of the robot without elastic coupling becomes larger as the joint damping coefficients 


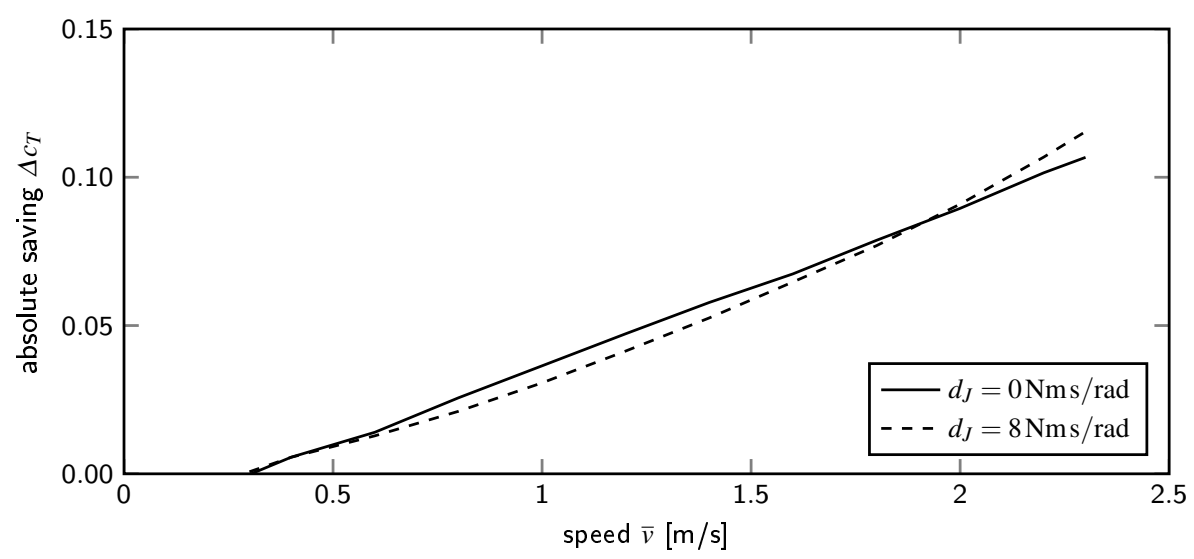

Fig. 23 Comparison of the absolute saving of the specific cost of transport $\Delta c_{T}$ by the elastic coupling with and without viscous joint damping

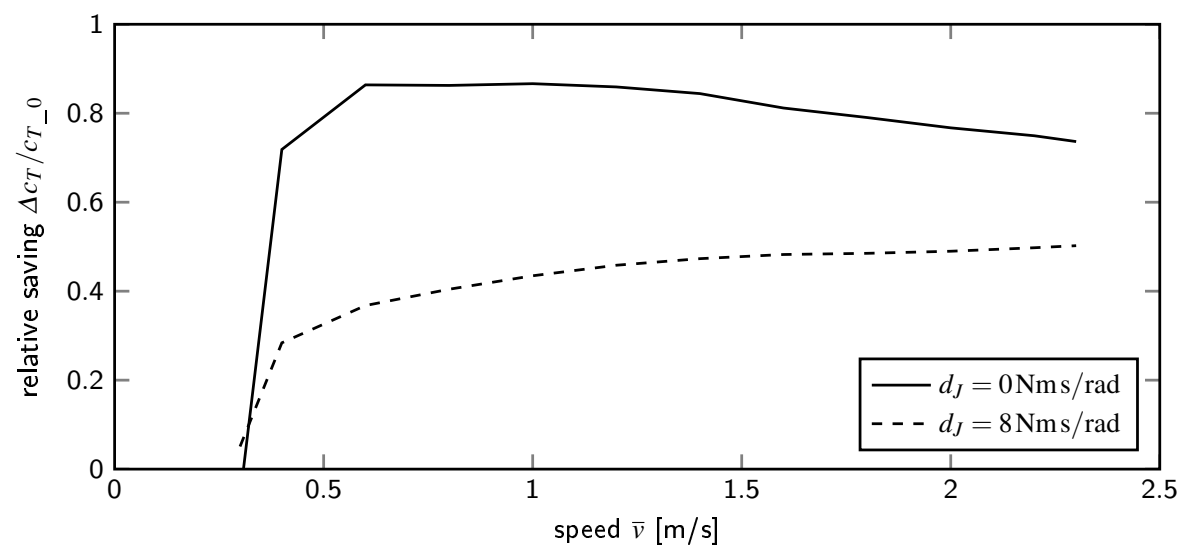

Fig. 24 Comparison of the relative saving of the specific cost of transport $\Delta c_{T} / c_{T_{-}}$by the elastic coupling for different joint damping coefficients $d_{J}$

$d_{J}$ increase while the absolute saving $\Delta c_{T}$ is constant, the relative saving of the specific cost of transport $\Delta c_{T} / c_{T \_0}$ decreases. For the assumed reduced drive train with joint damping coefficient $d_{J}=8 \mathrm{Nms} / \mathrm{rad}$ the mean relative saving or the reduction of the mean specific cost of transport $\bar{c}_{T}$ decreases from $80.1 \%$ to $47.0 \%$ and the reduction of the mean heat load or the mean specific static energy $\bar{e}_{\text {stat }}$ from $88.4 \%$ to $81.5 \%$. By the transition from the academic case without joint damping to a realistic value, the relative saving of specific cost of transport by elastic couplings decreases, however, it stays in a range relevant for practical application.

\subsection{Stability and sensitivity}

Optimization is always a specialization. This paper focuses on energy efficiency by optimizing the robot's elastic coupling as well as the motion. This raises the question if the increase 
in energy efficiency sacrifices stability and increases sensitivity with respect to disturbances of the motion. Through the use of input-output linearization, the dynamics of the robot are completely described by its hybrid zero dynamics. Hence, the stability and sensitivity of the robot's motion can be evaluated by analyzing the stability and sensitivity of the solution of the hybrid zero dynamics. The stability of the robot's motion is investigated by means of the absolute value of the Floquet multiplier of the Poincare map of the trajectory of zero dynamics just before the impact (cf. Eq. (61)). The sensitivity of the robot's motion is investigated by the relative size of the basin of attraction of the stable trajectory of the zero dynamics evaluated in the Poincaré map just before the impact (cf. Eq. (62)).

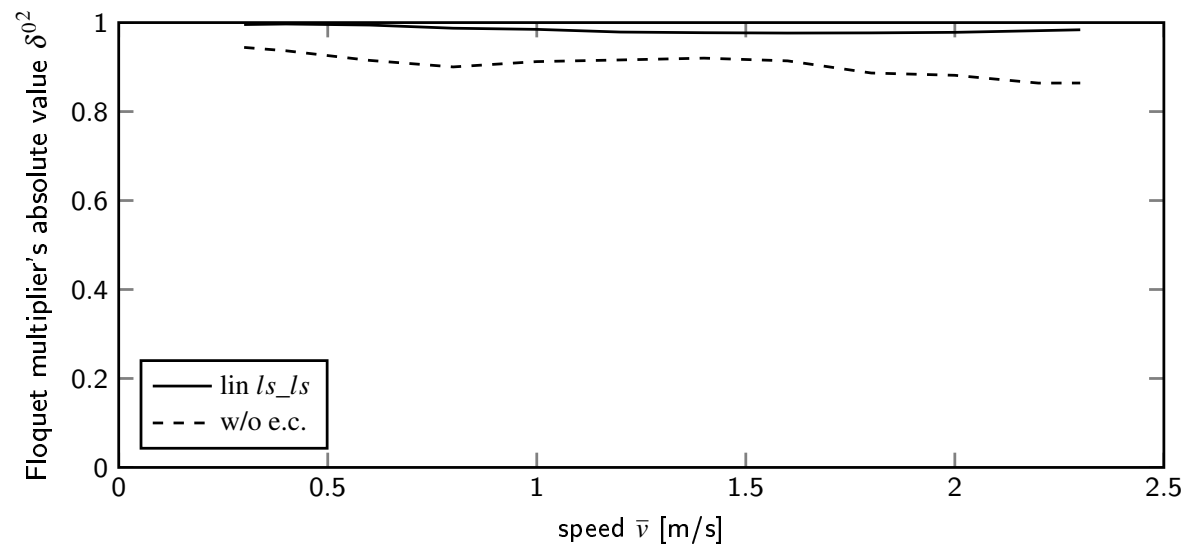

Fig. 25 Comparison of the Floquet multiplier's absolute value $\delta_{0}{ }^{2}$ for the robot with and without elastic coupling

Figure 25 shows the Floquet multiplier's absolute value $\delta_{0}{ }^{2}$ for the robot with and without elastic coupling. In both cases the absolute value of the Floquet multiplier is less than one. Accordingly, the solution and therewith the motion of the robot is stable. Since the reduction of specific cost of transport by elastic couplings is based on the reduction of impact loss, $\delta_{0}$ has to approach the value 1 (cf. Eq. (58)). Therefore, the Floquet multiplier of the robot with elastic coupling is greater than without elastic coupling. This results in a slower decay rate of disturbances in the total angular momentum and thus a slower transition to the desired average speed.

Figure 26 depicts the relative size of the basin of attraction $s_{\mathscr{B}}$ for the robot with and without elastic coupling. For low speeds $(\bar{v}=0.3-0.7 \mathrm{~m} / \mathrm{s})$ the relative size of the basin of attraction of the stable solution of the robot with elastic coupling is greater than without elastic coupling. For medium speeds $(\bar{v}=0.7-1.9 \mathrm{~m} / \mathrm{s})$ the relative size of the basin of attraction of the stable solution of the robot with elastic coupling is smaller than without elastic coupling and for high speeds $(\bar{v}=1.9-2.3 \mathrm{~m} / \mathrm{s})$ the relative sizes of the basins of attraction are approximately the same. The sensitivity of the robot's motion increases in general, however with the basin of attraction's minimal relative size of $84.6 \%$ it is high enough for practical usage at each considered speed.

The utilization of elastic couplings to reduce the specific cost of transport results in a motion with slightly slower convergence rate with respect to disturbances and slightly decreases the size of the relative basin of attraction. 


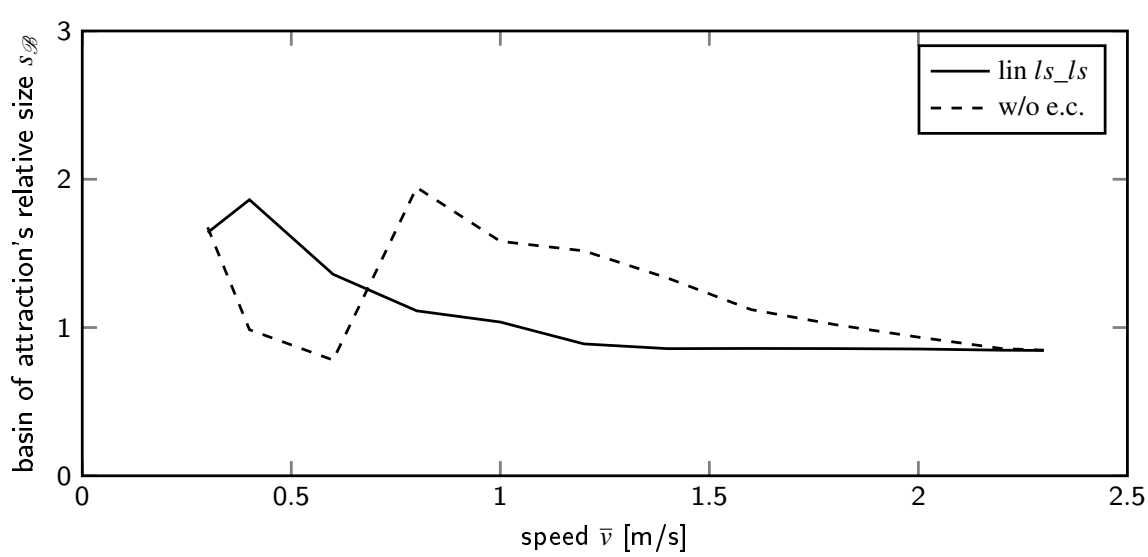

Fig. 26 Comparison of the basin of attraction's relative size $s_{\mathscr{B}}$ for the robot with and without elastic coupling

\subsection{Design proposal for the optimal elastic coupling}

Thus far the optimal elastic coupling in form of the elementary elastic coupling of the shanks between the legs $\left(l s_{-} l s\right)$ was merely described by its joint torque $\mathbf{T}_{l s \_l s}$ (cf. Eq. (83)). In this section a design of the optimal elastic coupling - which implements the two subfunctions elasticity and joint angle transmission via two subsystems - is proposed for planar as well as three-dimensional robots.

The design proposal for a planar robot in Fig. 27(a) and (b) consists of two belt drives and one tension spring. The subfunction joint angle transmission is realized by the belt drives, whereas the pulley coaxial with the knee joint is fixed to the shank and the pulley coaxial with the hip joint is pivoted. The ratio of the pulleys diameters defines the transmission ratio $i_{l s} l_{s}$ of the elastic coupling. The subfunction elasticity is realized by one simple tension spring between the pivoted pulleys. The degree of nonlinearity of the elastic coupling can be adjusted by the strain-stress curve of the material of the spring or the axial distance of the spring's fastening points on the pivoted pulleys.

The design proposal for a three-dimensional robot with spherical hip joints in Fig. 27(c) extends the planar design via Bowden cables to allow for independent spatial movement of both legs. The joint angle transmission is realized via belt drives as in the planar case. The elastic coupling of the pulleys is realized via linear tension springs and Bowden cables. The degree of nonlinearity can be adjusted by the strain-stress curve of the spring material or by the geometric arrangement of the Bowden cables with respect to the pulleys.

The suggested design of the optimal elastic coupling does not influence the motion of the trunk or the parallel motion of the shanks which is advantageous in manipulation tasks, it is primarily active during the walking motion. It can be realized with simple components and can be used to upgrade an existing robot.

\section{Conclusion and outlook}

It is known from literature [13] that a bipedal robot will only walk efficiently if its control does not fight against its natural dynamics but allows for it to evolve. For a given robot design elastic couplings are the only possibility to shape the natural dynamics. Hence, the aim of 


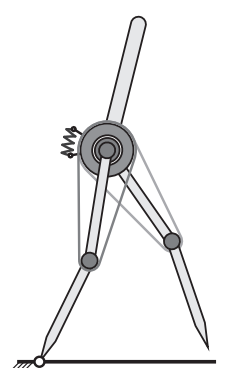

(a)

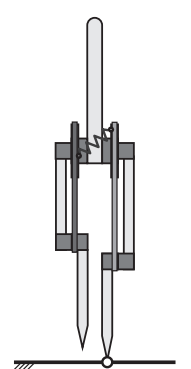

(b)

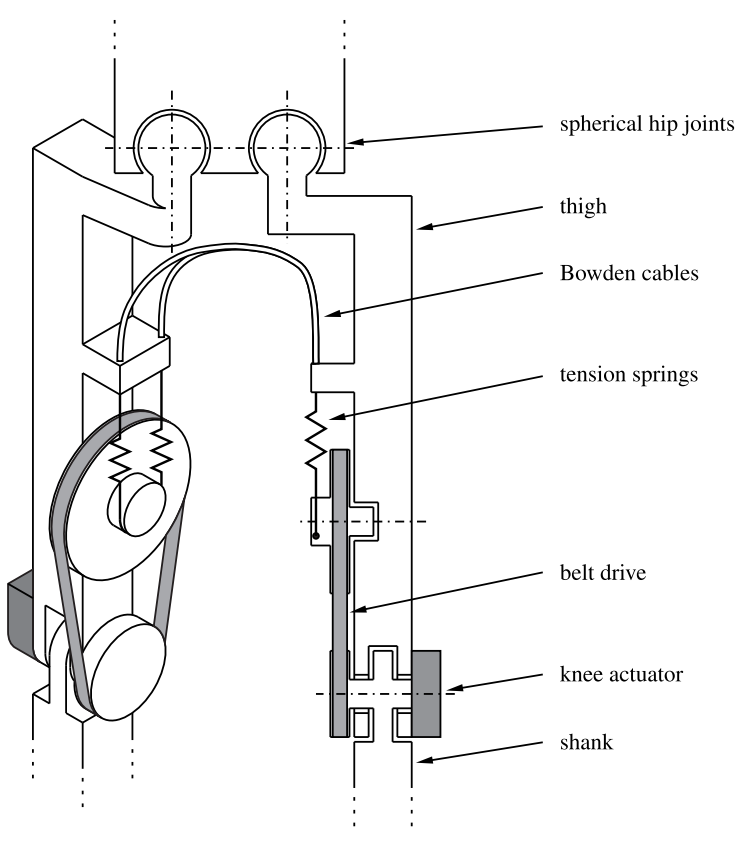

(c)

Fig. 27 Design proposal of the optimal elastic coupling. Side view (a) and rear view (b) of the design proposal for a planar robot consisting of two belt drives and one tension spring. Perspective and sectional views (c) of the design proposal for a robot with spherical hip joints consisting of two belt drives coupled via tension springs and Bowden cables

this paper was to investigate the influence of elastic couplings on the energy efficiency of a bipedal walking robot. For this purpose an $1.80 \mathrm{~m}$ tall, $80 \mathrm{~kg}$ heavy, underactuated robot was considered, feedback controlled via input-output linearization and analyzed in the hybrid zero dynamics as proposed by [44]. The gained knowledge is transferable to every bipedal robot provided that its control allows for the evolution of natural dynamics.

In order to quantify the energy efficiency and to evaluate the influence of the elastic couplings, the specific cost of transport was introduced as energy input divided by distance traveled and weight. The energy input was defined as the integral of the actuators' electric power intake during one step. For the development of an energy efficient robot an optimization process was designed, minimizing the mean specific cost of transport in the walking speed range $\bar{v}=0.3-2.3 \mathrm{~m} / \mathrm{s}$ by optimizing the motion and the elastic coupling of the robot simultaneously. The considered parameters of the elastic coupling are movement speed independent.

The energy turnover of the robot was analyzed in detail to explain the effect which reduces the specific cost of transport. For the robot without elastic couplings the energy input by the actuators is mainly dissipated by negative mechanical work during braking in the stance leg knee joint. The shock absorbing knee function reduces the energy loss of the plastic impact at touchdown in the double support phase. By modeling the robot during the double support phase as an inverted mathematical pendulum it could be shown that the reduction of step length is an alternative measure to reduce the impact loss. At a given desired speed, the reduction of step length directly increases the double step frequency. By 
modeling the swing leg during the single support phase as a physical pendulum it could be shown that the double step frequency of the robot without elastic coupling is far above the resonance frequency of the swing leg motion. Using the elastic coupling between the legs increases the swing leg motion's resonance frequency thus the effect of lower impact losses via smaller step lengths can be exploited. Using the optimized elastic coupling between the legs the robot moves in resonance over a broad range of speeds and the specific cost of transport is reduced.

The elastic couplings within each leg cannot be used optimally in the studied range of speeds, because the trunk inclination angle and the knee flexion angle depend on movement speed. Combinations of different elastic couplings between the leg did not show an improvement in the specific cost of transport. The optimal elastic coupling consists of the elementary elastic coupling of the shanks between the legs.

The optimal linear elastic coupling of the shanks reduces the mean specific cost of transport by $80.1 \%$ for the academic robot without joint damping and by $47.0 \%$ for the robot with realistic joint damping. While the relative reduction decreases with increasing joint damping, the absolute reduction is maintained.

The mean static electric energy, the heat load of the actuators, is reduced for the academic robot without joint damping by $88.4 \%$ and for the robot with realistic joint damping by $81.5 \%$ using a linear elastic coupling of the shanks. This allows for downsizing of the actuators and thus reduced weight and friction of the robot.

The optimization of the robot's motion and elastic coupling towards energy efficiency does not severely interfere with stability and sensitivity of the motion. Using elastic couplings the motion of the robot remains stable. However, the decay rate of perturbations decreases as a matter of the reduced impact loss. The robot's motion becomes slightly more sensitive due to the elastic couplings because the relative size of the basin of attraction of the stable solution decreases, but stays sufficiently large for practical application.

It can be summarized that by using elastic couplings the specific cost of transport of the robot can be reduced significantly with minor sacrifices in stability and slight increases of the motion's sensitivity with respect to disturbances.

The design proposal for the optimal elastic coupling for planar robots consists of two belt drives and one tension spring. The design proposal for the optimal elastic coupling for three-dimensional robots consists of two belt drives which are elastically coupled with Bowden cables. Neither one interferes with trunk or parallel shank motion of manipulation tasks and can be used to upgrade an existing robot.

In the future the effect of elastic couplings on a more realistic robot model with extended feet will be investigated. The increase in the number of rigid bodies results in further topologies of elastic couplings and therewith options to reduce the specific cost of transport. Furthermore, we plan to investigate the influence of elastic couplings on planar running gaits. However, the application of the presented method to three-dimensional robot models is not planned in the near future. Prior to the investigation of three-dimensional models, the aim is the validation of the effect of elastic couplings in a hardware experiment.

\section{References}

1. M. Ahmadi and M. Buehler. A control strategy for stable passive running. In Proc. 1995 IEEE/RSJ Int. Conf. Intell. Rob. Syst. (IROS), volume 3, pages 152-157, 1995.

2. R. M. Alexander. Three uses for springs in legged locomotion. Int. J. Rob. Res., 9(2):53-61, 1990.

3. F. Asano. Stability analysis of underactuated compass gait based on linearization of motion. Multibody Sys. Dyn., 33(1):93-111, 2015 
4. F. Bauer. Optimierung der Energieeffizienz zweibeiniger Roboter durch elastische Kopplungen. PhD thesis, Karlsruhe Institute of Technology, 2014.

5. F. Bauer, A. Fidlin, and W. Seemann. Energy efficient bipedal robots walking in resonance. ZAMM - J. Appl. Math. Mech., 94(11):968-973, 2014.

6. F. Bauer, H. Hetzler, A. Pagel, and W. Seemann. Do Non-linearities Enhance Stability of Bipedal Locomotion? In Proc. 2010 Int. Conf. Sim. Model. Program. Auton. Rob. (SIMPAR), pages 104-112, 2010.

7. P. A. Bhounsule, J. Cortell, A. Grewal, B. Hendriksen, J. D. Karssen, C. Paul, and A. Ruina. Lowbandwidth reflex-based control for lower power walking: $65 \mathrm{~km}$ on a single battery charge. Int. J. Rob. Res., 33(10):1305-1321, 2014.

8. E. Borzova and Y. Hurmuzlu. Passively walking five-link robot. Automatica, 40(4):621-629, 2004.

9. D. Budday, F. Bauer, and J. Seipel. Stability and Robustness of a 3D SLIP Model for Walking Using Lateral Leg Placement Control. In Proc. 2012 ASME IDETC, volume 4, pages 859-866, 2012.

10. J. Calsamiglia, S. W. Kennedy, A. Chatterjee, A. Ruina, and J. T. Jenkins. Anomalous frictional behavior in collisions of thin disks. J. Appl. Mech., 66(1):146-152, 1999.

11. C. Chevallereau, G. Abba, Y. Aoustin, F. Plestan, E. R. Westervelt, C. Canudas-de-Wit, and J. W. Grizzle. RABBIT: A testbed for advanced control theory. IEEE Control Syst. Mag., 23(5):57-79, 2003.

12. T. Chyou, G. F. Liddell, and M. G. Paulin. An upper-body can improve the stability and efficiency of passive dynamic walking. J. Theor. Biol., 285(1):126-135, 2011.

13. S. Collins, A. Ruina, R. Tedrake, and M. Wisse. Efficient bipedal robots based on passive-dynamic walkers. Science, 307(5712):1082-1085, 2005.

14. S. Cotton, I. M. C. Olaru, M. Bellman, T. van der Ven, J. Godowski, and J. Pratt. Fastrunner: A fast, efficient and robust bipedal robot. Concept and planar simulation. In Proc. 2012 IEEE Int. Conf. Rob. Auto. (ICRA), pages 2358-2364, 2012.

15. P. De Leva. Adjustments to Zatsiorsky-Seluyanov's segment inertia parameters. J. Biomech., 29(9):1223-1230, 1996.

16. J. C. Dean and A. D. Kuo. Elastic coupling of limb joints enables faster bipedal walking. J. Roy. Soc. Interface, 6(35):561-573, 2009.

17. J. M. Donelan, R. Kram, and A. D. Kuo. Mechanical work for step-to-step transitions is a major determinant of the metabolic cost of human walking. J. Exp. Biol., 205(23):3717-3727, 2002.

18. V. Duindam and S. Stramigioli. Optimization of Mass and Stiffness Distribution for Efficient Bipedal Walking. In Proc. 2005 IEEE/RSJ Int. Conf. Intell. Rob. Syst. (IROS), pages 18-21, 2005.

19. R. J. Full and D. E. Koditschek. Templates and anchors: neuromechanical hypotheses of legged locomotion on land. J. Exp. Biol., 202(23):3325-3332, 1999.

20. B. Gamus and Y. Or. Analysis of dynamic bipedal robot walking with stick-slip transitions. In Proc. 2013 IEEE Int. Conf. Rob. Auto. (ICRA), pages 3348-3355, 2013.

21. M. Garcia, A. Chatterjee, A. Ruina, and M. Coleman. The Simplest Walking Model: Stability, Complexity, and Scaling. J. Biomech. Eng., 120(2):281-288, 1998.

22. R. Ghigliazza, R. Altendorfer, P. Holmes, and D. E. Koditschek. A Simply Stabilized Running Model. SIAM J. Appl. Dyn. Syst., 2(2):187-218, 2003.

23. M. Gomes and A. Ruina. Walking model with no energy cost. Phys. Rev. E, 83(3):032901, 2011.

24. J. W. Grizzle, J. Hurst, B. Morris, H.-W. Park, and K. Sreenath. MABEL, a new robotic bipedal walker and runner. In Proc. 2009 Am. Control Conf. (ACC), pages 2030-2036, 2009.

25. M. Hirose and K. Ogawa. Honda humanoid robots development. Philos. Trans. R. Soc. London, Ser. A, 365(1850):11-19, 2007.

26. K. Kaneko, F. Kanehiro, S. Kajita, H. Hirukawa, T. Kawasaki, M. Hirata, K. Akachi, and T. Isozumi. Humanoid robot HRP-2. In Proc. 2004 IEEE Int. Conf. Rob. Auto. (ICRA), volume 2, pages 1083-1090 Vol.2, April 2004.

27. A. D. Kuo. Stabilization of Lateral Motion in Passive Dynamic Walking. Int. J. Rob. Res., 18(9):917930, 1999.

28. A. D. Kuo. A Simple Model of Bipedal Walking Predicts the Preferred Speed-Step Length Relationship. J. Biomech. Eng., 123(3):264-269, 2001.

29. S. Lohmeier, T. Buschmann, and H. Ulbrich. System Design and Control of Anthropomorphic Walking Robot LOLA. IEEE/ASME Trans. Mechatron., 14(6):658-666, 2009.

30. T. McGeer. Passive Bipedal Running. Proc. R. Soc. London, Ser. B, 240(1297):107-134, 1990.

31. S. Mochon and T. A. McMahon. Ballistic walking. J. Biomech., 13(1):49-57, 1980.

32. T. Narukawa, M. Takahashi, and K. Yoshida. Efficient walking with optimization for a planar biped walker with a torso by hip actuators and springs. Robotica, 29(04):641-648, 2011.

33. H.-W. Park, K. Sreenath, J. W. Hurst, and J. W. Grizzle. Identification of a Bipedal Robot with a Compliant Drivetrain. IEEE Control Syst. Mag., 31(2):63-88, 2011.

34. P. T. Piiroinen, H. J. Dankowicz, and A. B. Nordmark. Breaking Symmetries and Constraints: Transitions from 2D to 3D in Passive Walkers. Multibody Sys. Dyn., 10(2):147-176, 2003. 
35. F. Plestan, J. W. Grizzle, E. R. Westervelt, and G. Abba. Stable walking of a 7-DOF biped robot. IEEE Transactions on Robotics and Automation, 19(4):653-668, 2003.

36. G. Pratt. DARPA-BAA-12-52 - Maximum Mobility and Manipulation (M3) - Actuation. DARPA. https://www.fbo.gov (2012). Accessed 23 May 2013, 2012

37. K. Radkhah, T. Lens, and O. von Stryk. Detailed dynamics modeling of BioBiped's monoarticular and biarticular tendon-driven actuation system. In Proc. 2012 IEEE/RSJ Int. Conf. Intell. Rob. Syst. (IROS), pages 4243-4250. IEEE, 2012.

38. K. Radkhah, C. Maufroy, M. Maus, D. Scholz, A. Seyfarth, and O. von Stryk. Concept and design of the biobiped1 robot for human-like walking and running. Int. J. Humanoid Rob., 08(03):439-458, 2011.

39. M. H. Raibert. Legged robots that balance. MIT Press, 1986.

40. A. Ramezani, J. W. Hurst, K. A. Hamed, and J. W. Grizzle. Performance Analysis and Feedback Control of ATRIAS, A Three-Dimensional Bipedal Robot. J. Dyn. Syst. Meas. Contr., 136(2):021012, 2014.

41. U. J. Römer, C. Kuhs, M. J. Krause, and A. Fidlin. Simultaneous optimization of gait and design parameters for bipedal robots. In Proc. 2016 IEEE Int. Conf. Rob. Auto. (ICRA), 2016 - accepted.

42. W. Schiehlen. Energy-Optimal Design of Walking Machines. Multibody Sys. Dyn., 13(1):129-141, 2005.

43. C. M. Thompson and M. H. Raibert. Passive Dynamic Running. In Experimental Robotics I, volume 139, pages $74-83,1990$

44. E. R. Westervelt, J. W. Grizzle, C. Chevallereau, J. H. Choi, and B. Morris. Feedback control of dynamic bipedal robot locomotion. CRC Press, 2007.

45. M. Wisse, D. G. Hobbelen, and A. L. Schwab. Adding an Upper Body to Passive Dynamic Walking Robots by Means of a Bisecting Hip Mechanism. IEEE Trans. Rob., 23(1):112-123, 2007.

46. M. Wisse, A. L. Schwab, R. Q. van der Linde, and F. C. T. van der Helm. How to keep from falling forward: elementary swing leg action for passive dynamic walkers. IEEE Trans. Rob., 21(3):393-401, 2005 